\title{
Von der Hol- zur Bring-Bibliothek
}

\section{von Helge Steenweg*}

\section{Einleitung:}

Wenn im Titel dieses Aufsatzes die Begriffe Hol- und Bring-Bibliothek benutzt werden, so soll dies Veränderungen anzeigen, die sich auf unterschiedlichen Ebenen bemerkbar machen.

- Veränderungen im Selbstverständnis (Bibliotheken als Dienstleister)

- Veränderungen in der Bearbeitung und Vermittlung von Medien (Digitale Dokumente)

- Veränderungen im Benutzerverhalten (Der Nutzer holt sich die Informationen nicht mehr in der Bibliothek ab, sondern sie werden ihm an den Arbeitsplatz gebracht)

- Veränderungen in der technischen Darbietung (Pull-/Push-Prinzip; Der Nutzer kann entsprechend seinen Interessen in digitalen Bibliotheken gezielt Dokumente über Profile, Kanäle oder Agenten beziehen)

Der Titel soll eine Richtung beschreiben. Wir stehen derzeit in der Entwicklung der Bibliotheks- und Informationslandschaft größtenteils immer noch bei der Form der Hol-Bibliothek. Prognosen über die Zukunft abzugeben, dürfte beim derzeitigen Stand der Entwicklungen im Informationswesen schwer sein. Eine Möglichkeit der Weiterentwicklung dürfte in jedem Fall die Bring-Bibliothek darstellen. In diesem Aufsatz sollen einige Beschwernisse und Veränderungen dieser Entwicklung aufgezeigt und Vorschläge und Anregungen entwickelt werden, die mittelfristig in die Form einer Bring-Bibliothek münden. Auch das im Folgenden näher spezifizierte Projekt „Digibib-Hessen“ versteht sich nur als ein Baustein auf diesem Weg. Es muß nicht betont werden, daß die Holbibliothek noch sehr lange parallel existieren wird.

\section{Gekennzeichnet von Veränderungen}

\section{"Langer Weg.}

Die Zukunft des wissenschaftlichen Publikationswesens hat demnach gerade erst begonnen. Die größten Hindernisse zur Bewältigung der bevorstehenden Umwälzungen sind derzeit noch Angst, Trägheit und Tradition. Aber die Fortschreibung der alten Rollenverteilung in eine neue Umgebung ist immer nur ein allererster Schritt, bevor sich gänzlich andere Strukturen herausbilden. ${ }^{l}$

Dieser Schluß eines Artikels von Richard Sietmann beschreibt sehr deutlich die Zukunft und zugleich die gegenwärtigen Probleme, mit denen der Übergang ins digitale Publikationszeitalter verbunden ist. Welche zukünftige Struktur bzw. welche Arbeitsteilung zwischen Autoren, Fachgesellschaften, Fachinformationszentren, Bibliotheken und Verlagen herrschen wird, ist völlig offen.

Wenngleich man noch nicht ganz von einem "Kampf aller gegen alle"2 sprechen kann, ist doch allerorten eine große Unsicherheit über die eigene Zukunft zu verspüren. Die Erkenntnis, daß die immer schneller voranschreitende Technologie zu gewaltigen Umstrukturierungen führen wird, ist selbst traditionellen Vertretern klar. Im folgenden soll versucht werden, einige Aspekte aufzugreifen und sie aus einer Bibliothekssicht, die naturgemäß aus der eigenen Arbeit heraus

\footnotetext{
*Anschrift des Autors: Dr. Helge Steenweg, UB Kassel Informationsmanagement, Diagonale 10, 34127 Kassel.

${ }^{1}$ R. Sietmann, Zirkelspiele. Die wissenschaftliche Literaturversorgung steckt weltweit in der Krise, in: c’t 20, 1999, S. 216-231, hier S. 231; auch im WWW im c't-Archiv unter http://www.heise.de.

${ }^{2}$ R. Sietmann, Die Vertreibung aus dem Paradies, in Telepolis vom 12.1.2000, unter: http://www.heise.de/tp/deutsch/on/5672/1.html sieht ihn so: "Den Bibliotheken geht es um Besitzstandswahrung, den STM-Verlegern um den Erhalt attraktiver Erlöse, den Endbenutzern um die Sicherung des freien Zugangs zu den Ergebnissen der Forschung."
} 
stärker technisiert ist, zu gewichten. Wenn im Anschluß daran ein Pilotprojekt aus dem hessischen Multimedia-Programm "Hessen-Media" vorgestellt wird, dann ist dies der Versuch, einige theoretische Erkenntnisse und Diskussionen im Bereich der heutigen Bibliothek durch einen Prototypen in die Praxis zu übertragen.

\subsection{Quasi-Monopol der Bibliotheken ist verloren}

Seit Beginn der 90er Jahre läßt sich eine stetig schneller werdende Entwicklung auf dem Gebiet der Datenverarbeitung und Telekommunikation konstatieren. Sie führte nicht nur bei der sogenannten Hardware (Rechner- und Speichertechnologie) zu Veränderungen im Nutzungs- und Konsumverhalten. Insbesondere die Netz- und Kommunikationsfunktionalität hat innerhalb der letzten 10 Jahre Einzug in viele Lebensbereiche gefunden. Gerade im akademischen Bereich gehört das Internet zur völligen Normalität, liegen doch die Wurzeln u.a. im akademisch geprägten BITNET (Because It's Time NETwork) ${ }^{3}$.

Hielten die Bibliotheken über lange Zeit unverändert ein Quasi-Monopol für die Versorgung von Lehre und Forschung mit Studien- und Forschungsliteratur, die sie von den Verlagen als Produzenten von gedruckten und vereinzelt auch audiovisuellen Produkten erwarben bzw. lizensierten, so verbreiten mit zunehmender Bedeutung und Akzeptanz des Internets wissenschaftliche Autoren und Institutionen ihre Schriften und Daten auch eigenständig in Form von größtenteils frei zugänglichen Dokument-, PrePrint-oder Datenbank-Servern. Viele große wissenschaftliche Zeitschriftenverlage stellen mittlerweile ihre Produkte auf eigenen Servern zur Verfügung, so daß die Frage berechtigt ist, wo die zukünftige Stellung der Bibliothek anzusiedeln ist. Es ist nicht mehr damit getan, Geschäftsgänge zusammenzulegen und zu automatisieren oder ein DV-gestütztes Bibliothekssystem mit WWW-OPAC zu betreiben.

Gemessen an der kommerziellen Konkurrenz werden auch Institutionen der öffentlichen Hand immer mehr ihre Leistungen in Relation zu den Kosten setzen müssen ${ }^{4}$, wobei diese Problematik im öffentlichen Bereich aufgrund des geringen Handlungsspielraumes z.B. bei den Personalkosten schwer umzusetzen sein wird. Kostenmanagement und Budgetierung werden immer mehr zum Tragen kommen, da auf absehbare Zeit Etaterhöhungen illusorisch sind und zusätzliche Mittel nur über Sondermittel Bibliotheksetats verstärken werden.

\subsection{Verknappte Geldmittel}

In diesem Zusammenhang sich über sinkende Bibliotheksetats und Abbestellaktionen auszulassen, hieße Eulen nach Athen zu tragen. Selbst diejenigen Bibliotheken, die über einen stagnierenden Bibliothekshaushalt verfügen, treffen alljährlich entsprechende Abbestellmaßnahmen, da ihr Etat nominal durch die Preissteigerungen der Verlage und die

\footnotetext{
${ }^{3}$ Ursprünglich baute 1969 das amerikanische Verteidigungsministerium ein Computernetz für vier Computer auf, das sog. ARPANET (Advanced Research Projects Agency NET), das 1982 zum Internet ausgebaut und weiterentwickelt wurde. Aus dieser Zeit stammen die wesentlichen Bestandteile, das Transmission Control Protocol (TCP) und das Internet Protocol (IP), die namensgebend für die gesamte Protokollsammlung wurden. 1990 löste das vor allem aus amerikanischen Hochschulen bestehende, 1986 gegründete National Science Foundation Network (NSFNET) das ARPANET als wissenschaftliches Netzwerk ab.

${ }^{4}$ Arnoud de Kemp (Springer) hat in einem Interview im Februar 2000 bei R. Sietmann, Eine Zeitschrift auf den Server einer Universität zu legen, ist keine große Leistung, in: Telepolis vom 12.1.2000, auch unter: http://www.heise.de/tp/deutsch/inhalt/on/5673/1.html u.a. die These von Andrew Odlyzko, Wissenschafter bei AT\&T, referiert: "Das ist keine Vision, sondern eine ganz klare Schlussfolgerung aus der Kostenentwicklung namhafter Universitätsbibliotheken in Amerika. Hochinteressant! Er hat die Erwerbungsetats der Bibliotheken in den letzten Jahren mit der Entwicklung der Gebäude-, Personal- und Verwaltungskosten verglichen und dabei festgestellt, dass der Verwaltungsaufwand rapide angestiegen ist, die Erwerbungsetats dagegen kaum. Das beschreibt er als gefährliche Schere. Sie dokumentiert, dass die Bibliotheken sehr bürokratisch arbeiten, und durch ihre Verwaltung eigentlich eine Behinderung für die Grundversorgung mit Literatur und Information darstellen." - vgl. auch K. Ceynowa, A. Conners, Kostenmanagement für Hochschulbibliotheken, Frankfurt/M 1999 (ZfBB Sonderh. 76).
} 
Inflationsrate entsprechend betroffen ist. Unabhängig von irgendwelchen Diskussionen, wessen Verhalten zu welchem Ergebnis führt ${ }^{5}$, lohnt sich ein Blick vor allem nach Amerika, wo die Erwerbungspolitik in den Bibliotheken und die daraus resultierenden Folgen mehrfach untersucht wurden. Der Focus lag dabei weniger auf den Monographien als vielmehr auf den gerade auch für die Forschung in den naturwissenschaftlichen Fächern wichtigen Zeitschriften.

\section{Zeitschriftenkosten}

Ein Blick auf die Kostenentwicklung von Print-Zeitschriften aus den USA zeigt deutlich ein Mißverhältnis bei der Preisentwicklung von Zeitschriften gegenüber Monographien.

Während die Preise für Bücher in etwa dem allgemeinen Preisanstieg entsprechend stiegen, explodierten die Preise für wissenschaftliche Zeitschriften um das Dreifache innerhalb von 20 Jahren.

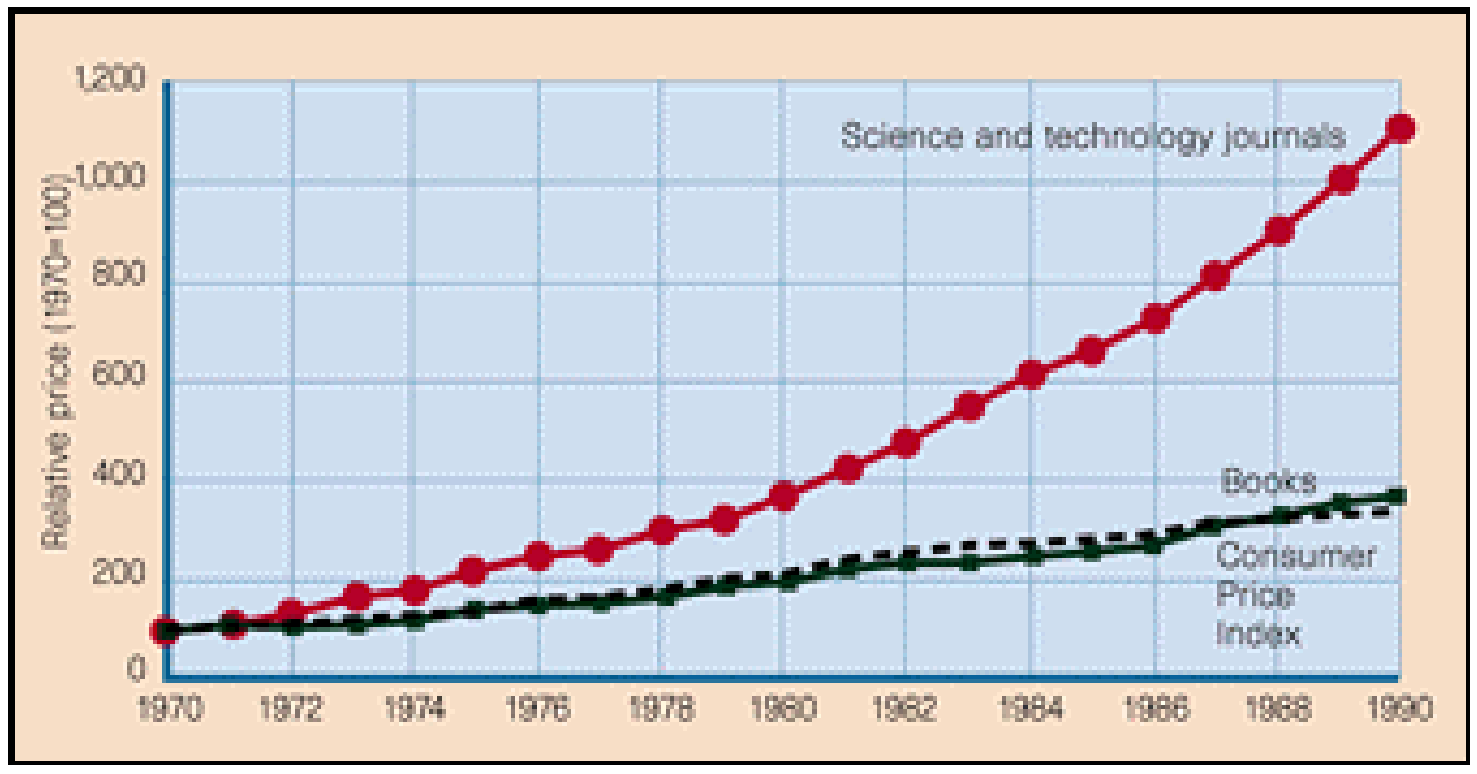

Mark DOBSON, The Writing is on the Web for Science Journals in Print, in: Nature 397, 1999, S. 195-200, hier: S. 197.

$\mathrm{Zu}$ einem ähnlichen Ergebnis kommen auch Untersuchungen der amerikanischen Association of Research Libraries (ARL):

\begin{tabular}{|lrr|}
\hline & $\begin{array}{r}\text { Prozentuale } \\
\text { Ausgabensteigerung } \\
\text { 1986-1998 }\end{array}$ & $\begin{array}{r}\text { Prozentuale jährliche } \\
\text { Ausgabensteigerung }\end{array}$ \\
Monographien & $33 \%$ & $2,4 \%$ \\
Gehälter & $78 \%$ & $4,9 \%$ \\
“operating"” & $86 \%$ & $5,3 \%$ \\
Zeitschriften & $152 \%$ & $8,0 \%$ \\
\hline
\end{tabular}

M. KYRILLIDON, Spending More for Less, unter: http://www.arl.org/newsltr/coll.html.

Der Durchschnittspreis stieg in dem Untersuchungszeitraum dreimal so stark wie die Inflationsrate. Konsequenzen aus dieser Entwicklung wird es mittelfristig mehrere geben. Die Abbestellaktionen in unseren Bibliotheken ist sicherlich eine sehr schmerzliche Erfahrung,

\footnotetext{
${ }^{5}$ Vgl. z.B. das Interview mit A. de Kemp (Springer) bei Sietman, Eine Zeitschrift (wie Anm. 4). Die Konferenz der Deutschschweizer Hochschulbibliotheken beschloß im Oktober 1999, Zeitschriften mit besonders markanten Preissteigerungen nicht mehr anzuschaffen - vgl. Sietmann, Die Vertreibung (wie Anm. 2).
} 
jedoch kann die Auswirkung dieser Entwicklung dadurch gemildert werden, daß Digitale Bibliotheks-Systeme durch die Bereitstellung digitaler Zeitschriften im Volltext Benutzern diese Funktionalität nicht nur erhält, sondern sie für die Forschung sogar effizienter (elektronische Suche, schnelle Bereitstellung, Bereitstellung direkt am Arbeitsplatz) macht. Eine zukünftige Aufgabe wird daher wohl auch darin bestehen, für Kauf- und Verhandlungsentscheidungen mit Verlagen seitens des Bibliotheksinformationsmanagements festzustellen, welche Zeitschriften intensiv genutzt werden ${ }^{6}$. Allerdings ist bei diesen Uberlegungen zu berücksichtigen, daß die Fachgebiete sich unterschiedlich in ihrer Nutzung und Ausprägung digitaler Informationen verhalten und auch deutlich anders forschungsmäßig orientiert sind.

\subsection{Nutzerverhalten}

Stellt man einmal die Zitationen zeitlich pro Fachdisziplin zusammen und ermittelt als sogenannte Halbwertzeit den Wert, an dem innerhalb eines definierten Zeitraumes die Hälfte der Zitationen erreicht werden, so ergibt sich als Graphik folgendes Schaubild:

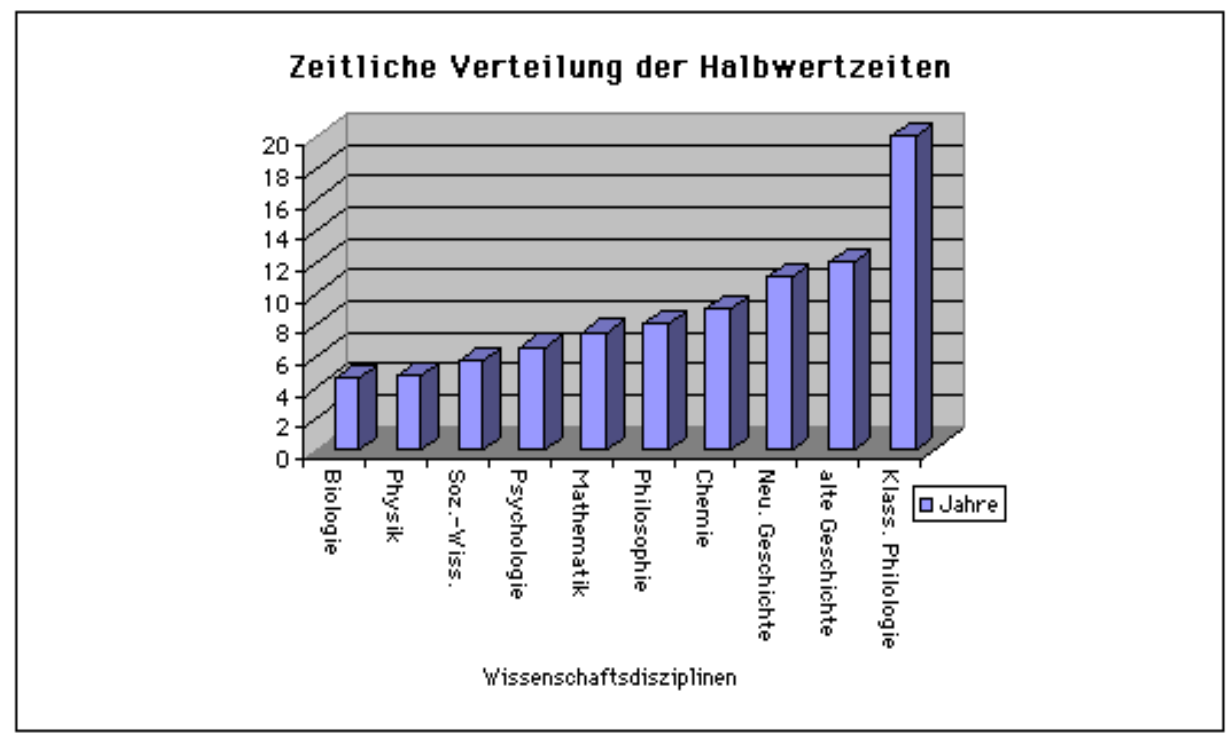

Deutlich treten die unterschiedlichen Rezeptionszeiten hervor, denen die verschiedenen Disziplinen unterliegen. Diese gilt es, bei der Beschaffung und Bewertung entsprechend zu berücksichtigen? ${ }^{7}$.

Entsprechend der Bedeutung von elektronischen Informationen haben sich besonders in den Naturwissenschaften frühzeitig Initiativen entwickelt, die eine möglichst unabhängige Form des wissenschaftlichen Informationsaustausches und Publizierens suchten und aufbauten. Es existieren mittlerweile etablierte fachbezogene Preprint-Server. Weiterentwicklungen sehen vor, die Fachbereichs-Server weltweit mittels Robot-Systemen ("Gatherer" zum Sammeln, "Broker" zum Indizieren) zu miteinander verbundenen Informationssystemen zusammenzuschließen. Dieser interessante Ansatz steht und fällt jedoch mit dem Engagement der Beteiligten. Nur wenn die Autoren sich bereit finden, die für die so automatisierte Zusammenstellung und Indizierung notwendigen strukturierten Formate und Metadaten selbständig zu liefern, ist diese Vorgehensweise erfolgreich. Interessanterweise vollzieht sich derzeit auch bei den Befürwortern der PrePrint-Server eine Entwicklung, die erkennt, daß für den Prozeß einer wissenschaftlichen

\footnotetext{
${ }^{6}$ Bei der Intepretation von Zugangsstatistiken ist eine gewisse Vorsicht gegeben, da technisch die Nutzung von Cache- oder Proxy-Servern eine derartige Statistik verändert.

${ }^{7}$ Ein Blick auf die zukünftige Rolle der Bibliothek auch aus geisteswissenschaftlicher Sicht bei : J. Mittelstrass, Der Bibliothekar als Partner der Wissenschaft, in: ZfBB 47/3, 2000, S. 243-253.
} 
Veröffentlichung nicht nur der Publikationszyklus mit seinen unterschiedlichen Stadien entscheidend ist, sondern auch die Archivierung dazu gehört. Anläßlich einer Tagung in Santa Fe, New Mexico, im Oktober 1999 gab es eine Vereinbarung, die neben der Definition einer gemeinsamen Metadatenstruktur eine gemeinsame Suchmaschine ("end user service") über die "archive server" zu einem "Universal Preprint Server" (UPS) vorsieht".

Diese aus den Fachbereichen entstandenen und von den Fachgesellschaften geförderten Initiativen zeigen zum einen die Diskussion über die Kommerzialisierung von Informationen im wissenschaftlichen Publikations- und Informationswesen an, sie weisen jedoch auch deutlich den Wunsch der Wissenschaftler aus, die Möglichkeiten der neu zur Verfügung stehenden Medien nutzen zu können. Es ist mittlerweile eine Binsenwahrheit, daß der Nutzer seine benötigten Informationen sofort an seinem Arbeitsplatz präsentiert bekommen will. Er möchte natürlich in Metadatenbanken Literatur recherchieren können, diese jedoch auf Mausklick anschließend direkt im Volltext auf dem Monitor lesen können. Ein Zusatzservice wäre sicherlich, wenn der Nutzer zudem ähnliche Dokumente auf Anforderung vom System präsentiert bekäme (Relevanzrückkopplung) oder aber dem System-Agenten mitteilen könnte, über welche Dokumente er in der nächsten Zeit unterrichtet werden möchte (Agent-Funktionalität). Bei einer Analyse des nutzerbezogenen Accountings des WIN/Internet-Gateways wird man an vielen Hochschulstandorten feststellen, daß der Anteil des Telefongateways (PPP-Zugänge, eingehender Datenverkehr) in den letzten Jahren erheblich zugenommen hat ${ }^{9}$. Dies bleibt natürlich nicht ohne Konsequenzen für Bibliotheken. Wenn die Benutzer zu einem gewissen Teil an ihren Arbeitsplätzen oder Zuhause recherchieren und/oder ausleihen möchten, dann muß mit Fachleuten ein entsprechendes Angebot auf- resp. ausgebaut werden. Es heißt allerdings auch, daß sich der Ausbau mit eigener PC-Infrastruktur für Benutzer am Ort an dem jeweiligen Nutzungsgrad orientieren muß - eine Maßnahme, die sich aufgrund andersgearteter Auslastungen und Nutzungsverhalten vor einigen Jahren gänzlich anders darstellte.

Im Vordergrund wird vielmehr eine passende Netzinfrastruktur stehen. In Zusammenarbeit mit den Bibliotheken werden die Hochschulrechenzentren resp. Netzzentren für eine den Anforderungen angemessene physikalische Netz- und Informationsinfrastruktur sorgen müssen. Möchte man beispielsweise Multimedia-Anwendungen und interaktive Lehrangebote über das Internet anbieten, so sind schnelle und sichere Datennetze bis in den tertiären Sektor notwendig, selbst wenn derzeit die Kosten sicherlich manche dieser Ansprüche ausbremsen.

\subsection{Neue Aufgaben als Informationsvermittler}

Sehr deutlich hat sich im Bibliothekswesen in den letzten Jahren aufgrund der veränderten Finanzen und Publikationsformen eine Neuorientierung bei den Aufgaben ergeben. Gegenüber der früher stark betonten Bestandsorientierung hat die Beschaffungs- oder Nachweisorientierung stark an Bedeutung gewonnen ${ }^{10}$. Dabei geht es vorrangig um Beschaffung, Vermittlung und Archivierung elektronischer Medien. Da vielfach rechtliche Beschränkungen in Form von Copyright oder auf benutzerspezifische Gruppen (nur Campus, Fachbereich etc.) existieren, bedarf es einer Organisations- und Vermittlungsstelle, um diese Informationen für Nutzer adäquat aufzubereiten. Zum Erwerb von Büchern und Zeitschriften tritt als weitere Aufgabe der Umgang mit Nutzungslizenzen und Zugangsrechten.

Dabei bedarf auch die Form und Präsentation einer entsprechenden Dienstleistung. "Eine Zeitschrift auf den Server einer Universität zu legen, ist keine große Leistung" "ll. Pointierter läßt es sich wohl kaum ausdrücken. Es kann also nicht nur darum gehen, an jedem Standort

\footnotetext{
${ }^{8}$ H. van de Sompel, C. Lagoze: The Santa Fe Convention of the Open Archives Initiative, in : D-Lib Magazine 6/2, 2000, http://www.dlib.org/dlib/february00/vandesompel-oai/022vandesompel-oia.html; H. van de Sompel, Th. Krichel, M.L. Nelson: The UPS Prototype: An Experimental End-User-Service across E-Print Archives, in: D-Lib Magazine 6/2, 2000, http://www.dlib.org/dlib/february00/vandesompel-ups/022vandesompel-ups.html.

${ }^{9}$ Nach Auskunft des HRZ an der GH Kassel beträgt der Anteil derzeit über 50\% - frdl. Auskunft von Dr. R. Henze.

${ }^{10}$ A. Keller, W. Neubauer, Dienstleistungsangebote von Bibliotheken in elektronischer Form, in: NfD 50, 1999, S. 407-412, hier: S. 408.

${ }^{11}$ Ausspruch von Arnoud de Kemp bei R. Sietmann, Eine Zeitschrift (wie Anm. 4).
} 
vereinzelte elektronische Dokumente im Volltext ohne ein Retrieval als "elektronische Bibliothek" zu titulieren oder vor Ort einige auf spezielle Bedürfnisse ausgerichte Perl-Scripts als Suchmaschine zu definieren, da bereits professionelle Metasuchmaschinen, wie MetaGer oder KVK existieren.

Es muß zudem die Arbeit der Konsortien verstärkt werden - gerade auch im Hinblick auf eine Koordinierung der Abbestellungen (z.B. von gedruckten Mehrfachexemplaren), die ihrerseits für eine überregionale (verteilte oder zentrale) Erschließung der erworbenen Informationen sorgen sollte. Der Terminus "erworben" zeigt allerdings auch eine Problematik auf, die es zu beachten gilt. In der Regel erwerben Bibliotheken bei elektronischen Zeitschriften, um die es in der Regel geht, nur einen zeitlich und benutzerspezifisch beschränkten Zugang zu den betreffenden Daten. Dies kann z.B. ein Jahrgang einer Zeitschrift sein, der zukünftig ohne Zeitbeschränkungen recherchiert werden kann, oder der elektronische Zugang zu einer Zeitschrift befristet auf den jeweiligen Vertragszeitraum.

Der Erwerb von wie auch immer limitierten Nutzungsrechten bedeutet einen nicht unwesentlichen Einschnitt im bisherigen Aufgabenspektrum. Bislang kaufte die Bibliothek ein Publikationsprodukt, besaß es also, und stellte es zur Verfügung. Das Buch wurde, wie etymologisch das Wort Bibliothek aussagt, in das Bücher-Regal gestellt. Durch den Kauf von Zugangsrechten zu einem Server wird die bisherige Aufgabe, zur Archivierung und Bereitstellung von Informationen tätig zu sein, massiv erschwert, sofern nicht Möglichkeiten gefunden werden, dem entgegenzuwirken. Eine derartige Variante besteht zum einen darin, schon allein aus netztechnischen Geschwindigkeitsgründen - einen selbst betriebenen MirrorServer aufzusetzen. Eine andere Variante stellt ein überregionaler Dokumentenserver dar, der die zusätzlich erworbenen Archivkopien (Backup-Dateien) aufnimmt, und diese möglichst über ein Volltextretrieval für den jeweils berechtigten Nutzerkreis bereit stellt. Natürlich ist es derzeit, vor allem wenn das Personalproblem hinzugenommen wird, viel einfacher, den verlagseigenen Server über eine WWW-Seite bekannt zu machen oder mittels eines Scripts zu befragen. Dies ist jedoch schon allein aufgrund der Vielfalt und der unterschiedlichen technischen Realisierungen der betreffenden Verlagsserver in der Gesamtschau nicht immer nutzerfreundlich zu nennen. Ein eigener Archivbestand hingegen stellt neben den möglichen ausgefeilten Retrievalfunktion über alle Daten mittelfristig zudem eine gewisse Sicherheit gegen mögliche Verlagsverkäufe, Veränderungen in der Verlagspolitik, Konkurse etc. dar.

\subsection{Verlage}

Innerhalb der bisherigen Verteilung zwischen Autoren - Verlag - Bibliothek gab es über Jahrzehnte kaum Mißstimmungen, da die Aufgaben umrissen waren. Finanzielle Engpässe der Bibliotheken und die schnell voranschreitende Kommunikations- und Publikationstechnologie werden jedoch strukturelle Veränderungen bewirken, die derzeit allerdings nicht sicher zu prognostizieren sind. Arnould de Kemp hat, auf die zukünftige Arbeitsteilung zwischen Autoren, Fachgesellschaften, Fachinformationszentren, wissenschaftlichen Bibliotheken und Verlagen angesprochen, geantwortet: "Die Frage läßt sich kaum in einem Satz beantworten. Im Moment hat jeder seine Rolle, aber keiner ist damit so recht zufrieden. Ich denke, dass das Zusammenspiel im Prinzip darauf aufbaut. Große Brüche kann sich keiner leisten. Die bibliographischen Datenbanken und die Fachinformationszentren werden nicht obsolet werden; sie werden sich mit dem Volltext vernetzen und zum Teil ihr Material nicht mehr selbst erfassen, sondern von kommerziellen Verlagen bekommen." 12 Diese Antwort beschreibt aus Verlagssicht die Situation sehr treffend. Dennoch stellt sich die Frage, welche Aufgabe dann zukünftig noch den Bibliotheken zukommen kann. Nun ist deutlich, daß der Anteil gedruckter Informationen in den nächsten Jahren weiterhin gegenüber digitalen Produktionen höher zu veranschlagen sein dürfte, jedoch kann dies nicht heißen, daß man nicht gleichzeitig auch in den Bibliotheken größere Anstrengungen unternimmt, um von den kommenden strukturellen Veränderungen nicht überrannt zu werden.

\footnotetext{
${ }^{12}$ R. Sietmann, Eine Zeitschrift (wie Anm 4).
} 
Abgesehen von den in den letzten Jahren deutlich sich abzeichnenden

Verlagszusammenschlüssen und -aufkäufen und der verlagsinternen Umstellung auf neue

Publikationstechniken haben sich auch in den Beziehungen zwischen Bibliotheken und Verlagen deutliche Veränderungen ergeben. Viele große Zeitschriftenverlage bieten mittlerweile eigene Verlagsserver für ihre elektronischen Zeitschriften an oder bieten, da sie erkannt haben, daß der Zugriff auf weit entfernte Server mit entsprechender Netzlast mitunter unbefriedigend sein kann, von Tochterfirmen eigene Serversoftware für Mirrorserver an.

Auf die Wünsche der Nutzer nach elektronischen Varianten haben die Verlage reagiert, allerdings mit sehr unterschiedlichen Lizensierungsvarianten. Auch hier sind Änderungen erkennbar. War oftmals früher die Netz- an die Papierversion gekoppelt, so zeichnet sich allmählich ein umgekehrter Trend ab. Basis für Berechnungsmodelle ist die Onlineversion, die Papierversion kann gegen Aufpreis zusätzlich geordert werden. War früher der Wunsch nach einer Ausweitung der Lizenzen deutlich erkennbar, kann man heute bei Verhandlungen eher von einer Besitzstandswahrungspolitik reden. Diese Verträge sehen vor, daß innerhalb eines Konsortiums zwar abbestellt werden kann, jedoch muß die Vertragssumme als solche erhalten bleiben. Dies bietet allerdings für Bibliotheken auch eine Chance, kann man auf diese Weise die Zahl der Online-Zeitschriften durch die Abbestellung von Mehrfachexemplaren und Neubestellung von noch nicht vorhandenen Zeitschriften erhöhen. Allerdings bedingt diese Vorgehensweise möglicherweise einen nicht unerheblichen Zwang zur wirklichen Zweckgemeinschaft - mitunter ein Drahtseilakt, der auf Grund der jeweils autonomen Hochschulpolitik an jedem Standort nicht immer leicht ist.

Hauptkritikpunkt stellen - je nach Standpunkt - die Preissteigerungen bzw. Abbestellungen dar, die sich in einem steten Kreislauf befinden. Information wird immer stärker kommerzialisiert.

Die Generierung der DOI (Document Object Identifier, siehe Kap. 3.6) mit der Verknüpfung von Metadaten und Volltexten stellt einen deutlichen Schritt in diese Richtung dar, da der Verlag bei jedem Link in der Datenbank der DOI-Foundation festlegen kann, ob die dahinterstehende Information resp. das Lesen des Volltextes zu bezahlen oder frei zugänglich ist. Wenn, wie derzeit noch üblich, sowohl gedruckte als auch elektronische Varianten einer Zeitschrift produziert werden, ist einsichtig, daß diese (zusätzliche) Arbeitsweise finanziert werden muß. Es wird aber mit Interesse zu beobachten sein, wie sich die Preispolitik bei nur noch elektronisch produzierten Zeitschriften gestalten wird, wenn abgesehen vom eigentlichen Herstellungsprozeß veränderte Produktions-, Druck- und Verteilungsmechanismen gelten.

\section{Folgen und Möglichkeiten}

\subsection{Veränderungen in der Bibliothek}

Die große Problematik der Bibliotheken besteht derzeit darin, daß sie auf die vorstehenden Strukturänderungen nur schwer reagieren resp. reagieren können. Bei der heutigen Hochschulpolitik lastet auch auf der Bibliothek wie auf jeder anderen Hochschulinstitution ein Legitimationsdruck. Die Hochschulrahmengesetzgebungen einiger Länder sprechen bei der Informationsvermittlung an Hochschulen zukünftig nicht mehr dezidiert von Bibliothek, Rechenzentrum und Medienzentrum, sondern betiteln dies oftmals als Informationsmanagement und stellen dessen reale Organisation in die Politik der jeweiligen Hochschulspitze. Das derzeitige Bild ist nicht immer von der notwendigen Kooperationsbereitschaft geprägt; Konkurrenzdenken setzt ab und an dort ein, wo Synergie gefordert ist.

Die neuen Aufgaben, die auf universitäre Informationsvermittlungsstellen zukommen, ersetzen allerdings nicht die derzeit bestehenden Aufgaben; sie kommen zusätzlich hinzu. Es ist über eine lange Übergangszeit der große Bestand der gedruckten Literatur weiter zu pflegen und auszubauen, da auch die wissenschaftliche Neuproduktion zunächst überwiegend in herkömmlicher Form hergestellt werden wird. Die neuen Medien und die daraus erwachsenden Veränderungen und Chancen müssen zusätzlich be- resp. verarbeitet werden. Da dies realistisch betrachtet bei nominell sinkenden Etats vonstatten gehen soll, ist die Ausgangssituation nicht einfach. 


\section{Arbeitsabläufe}

Obwohl die meisten Bibliotheken über DV-gestützte Katalogisierungs- und Ausleihverfahren, CD-ROM-Server und Internet-Zugänge verfügen, ist der Weg zu einer Digitalen Bibliothek noch weit. In vielen Bereichen ist allerdings der Wandel bereits bei der Alltagsarbeit spürbar. Die Erwerbung umfaßt heute nicht mehr nur den Kauf von Büchern, Zeitschriften und CD's, sondern es werden auch Nutzungslizenzen und Zugangsrechte zu elektronischen Ressourcen erworben. Bei sinkenden Erwerbungsetats muß sich der Personalstand an den Käufen orientieren. In vielen Verbundsystemen sind bereits Schnittstellen und automatisierende Software vorhanden, um Käufe bei Produzenten oder beim Buchhandel vollends elektronisch abzuwickeln. Entsprechend verzeichnet die Katalogisierung nicht mehr nur neuerworbene gedruckte Bestände, sondern katalogisiert im zunehmenden Maße auch elektronische Medien, verknüpft Metadatensätze mit zugehörigen Volltexten und überprüft die Recherchefähigkeit von Suchmaschinen. Hierzu wird in einem verstärkten Maße DV-Wissen in Form von Datenbankkenntnissen, Übernahme von Datensätzen, Datenformaten und Verknüpfungsmechanismen von Datensätzen wichtig werden. Dies erscheint auf den ersten Blick einfacher als es ist, denn man hat es oftmals mit inhomogenen Datensätzen zu tun, z. B. bei der digitalen Retro-Katalogisierung von Zettelkästen. Auf der einen Seite existiert eine gepflegte Metadatenbank (z.B. Verbundkatalog), auf der anderen Seite eine per OCR erschlossene gescannte Bild- und Textdatenbank der Zettelkatalogisate. Eine direkte Verknüpfung beider Datenquellen ist meistens ausgeschlossen und in der Regel nur über eine übergeordnete Suchmaschine mit entsprechend optimierten Retrieval zu bewältigen.

Entsprechend der Zunahme an Nutzern über das Telefongateway und der Zunahme an elektronischen Medien wird sich die Ausleihe an Ausleihtheken zurückentwickeln. Auch die Nutzung von Selbstverbuchungsterminals, sofern sie in Freihandbeständen einsetzbar sind, spricht eine deutliche Sprache. Über eine automatisierte Fernleihe mit postalischer Lieferung der angeforderten Medien an den Arbeitsplatz resp. eigenen Haushalt ergeben sich weitere Veränderungen.

Da die neuen Medien sich jedoch auch arbeitsintensiv gestalten, wird gerade im Bereich der Nutzerbetreuung ein erhöhter Personalbedarf zu verzeichnen sein (s.u. Qualifizierung). Ein weites Aufgabenfeld erschließt sich über die Bearbeitung und Archivierung von elektronischen Volltexten. Neben der adäquaten Präsentation über ein leistungsfähiges Retrievalsystem, das betreut werden muß, müssen die gelieferten oder erworbenen Texte konvertiert, katalogisiert und in das System eingebunden werden. Realtiv selten sind bislang Bild-, Audio- oder Video-Daten, die gleichsam im Rahmen der multimedialen Betreuung in diese digitale Bibliothek aufgenommen und recherchefähig gemacht werden müssen.

Ohne zu sehr verallgemeinern zu wollen, muß gesagt werden, daß gerade auch bedingt durch den eingeschränkten Gestaltungsspielraum Umstrukturierungen nur langsam vonstatten gehen und das Bewußtsein, an einer wichtigen Schwelle zu stehen, die auch die bisherige Arbeit des Einzelnen massiv verändern wird, nicht immer vorhanden ist bzw. verständlich gemacht wird.

\section{Qualifizierung des Personals}

Damit ist ein sehr zentraler Aspekt bei dem Veränderungsprozeß angesprochen - Personal und Qualifikation. Bibliothekare arbeiten in einer festumrissenen und reglementierten Arbeitsumgebung, die sich über lange Jahre nur wenig verändert hat. Die mittlerweile schnell voranschreitenden technischen Veränderungen führen allerdings zum Teil zu Verunsicherungen, die es durch qualifizierte Fortbildungsmaßnahmen abzubauen gilt. Dabei kann es nicht darum gehen, aus Bibliothekaren technisch versierte Systemspezialisten zu machen, sondern es sollte vielmehr als Grundlage für weitere Entwicklungen die Bereitschaft und das Verständnis für neue Systeme und Strukturen vermittelt werden. Für den technischen Betrieb bedarf es entsprechender Fachleute, die es (trotz derzeitig schlechter Marktlage) einzustellen oder durch

Kooperation/Zusammenschluß mit anderen Hochschuleinrichtungen einzuwerben gilt.

Durch integrierte Geschäftsgänge, zukünftige Aufgabenverschiebungen und Automatisierung von Arbeitsabläufen werden Arbeiten reduziert und letztlich Stellen abgebaut werden. Die Betreuung 
der durch die neuen Medien entstehenden Aufgaben erfordern andererseits zusätzlich hochqualifizierte Arbeitskräfte, die es in den Bibliotheken zu qualifizieren gilt.

Neben den o.a. veränderten Arbeitsabläufen ist insbesondere auch an einen erhöhten Bedarf an Personal bei der Nutzerbetreuung zu denken, da die Nutzungsform neuer Medien sich sehr viel arbeitsintensiver gestalten wird, als dies derzeit bei herkömmlichen Medien der Fall ist. Allerdings wird auch die Form der Nutzerbetreuung sich viel stärker am Medium orientieren, denkbar sind z.B. Online-HelpDesk, Mail-Hotlines oder Call-Center.

\subsection{Organisationsveränderungen in der Universität}

Die neuen Publikationsformen in der wissenschaftlichen Informationsversorgung bedingen auf Dauer auch andere Organsisationsformen. Hatten die Bibliotheken bislang häufig nur mit gedruckten und vereinzelt audiovisuellen Medien zu tun, so gilt es jetzt, auch die sich immer mehr verstärkenden multimedialen Lern- und Lehreinheiten einzubeziehen. Hierzu bedarf es verstärkter Kooperation zwischen den Dienstleistungszentren Hochschulrechenzentrum, Bibliothek und Medienzentrum. Inwieweit sich diese Kooperation lokal zu Zusammenschlüssen dieser Einheiten oder Synergiezentren entwickelt, ist zum einen eine politische, zum anderen aber auch eine personelle Frage. Auf längere Sicht ist aus funktionaler und finanzieller Sicht ein Informationsmanagement für die jeweilige Hochschule als Einheit sinnvoll.

Bereits jetzt ist in zweischichtigen Bibliothekssystemen eine Tendenz zur Zentralisierung zu beobachten, die z.T. als "funktionale Einschichtigkeit" bezeichnet wird; in einschichtigen Systemen ist sie in der Regel per se vorhanden. Finanzielle Verteilkämpfe bestimmen nicht nur bei Bibliotheksetats manche Diskussion, auch bei der Beschaffung von digitalen Informationen, die aus zentralen Mitteln bezahlt werden, wird über entsprechende Fächer-Aufteilungsschlüssel zu diskutieren sein. Auch bei der Netznutzung könnte die Umstellung der DFN-EntgeldVereinbarung zu einer volumenbasierten Abrechnung in absehbarer Zeit nicht nur zur Einführung von Zwangs-Proxies sondern auch zu Kostenverteilmodellen führen.

Bei sich ständig verringernden Geldmitteln rücken immer stärker betriebswirtschaftliche Fragestellungen und Methoden ins Blickfeld der Universitäten. So werden im Rahmen der Prozeßkostenanalyse die Kosten bibliothekarischer Dienstleistungen ebenso eruiert werden wie auch eine ausgewogene Budgetierung zwischen Sach- und Personalmitteln deutlich an Wichtigkeit gewinnt. Dies wird sich in der Tendenz sicherlich noch verstärken, so daß in absehbarer Zeit interne Verrechnungssysteme zwischen Uni-Verwaltung, Fachbereichen und Dienstleistungszentren etc. nicht auszuschließen sind.

\subsection{Veränderungen im Verbund}

Immer wichtiger wird, da die einzelne Bibliothek nicht mehr jede gewünschte Information selbst vorrätig halten oder erwerben kann, der Zusammenschluß zu Konsortien. Dies führt sicherlich zu Preisvorteilen, bedingt jedoch auch eine größere Koordination und einen Zwang, im Sinne eines Kompromisses möglicherweise auch einmal gegen die eigenen Interessen zu stimmen. Keine leichte Aufgabe, wenn man bedenkt, daß ausdrücklich in vielen neuen Hochschulrahmengesetzgebungen eine größere Autonomie für die jeweilige Hochschule gewollt ist.

\subsection{Veränderungen bei den Autoren}

Bei den mittlerweile zur Verfügung stehenden Möglichkeiten zum wissenschaftlichen Publizieren wird den Autoren selbst auch ein nicht unwesentlicher Part zukommen. Wissenschaftliches Publizieren bedingt die Einhaltung bestimmter Regularien, digitales wissenschaftliches Publizieren erfordert darüber hinaus das Einhalten bestimmter Formate und Absprachen, wie die Bereitstellung von strukturierten Informationen im XML- oder SGMLFormat sowie die Lieferung von Metadaten zu der Publikation, um eine maschinelle, strukturierte 
Erfassung zu ermöglichen ${ }^{13}$. Hier wird ein nicht zu unterschätzendes Betätigkeitsfeld für zukünftige Informationsvermittler oder -manager an der Hochschule liegen: Die Schulung und Unterstützung von Wissenschaftlern beim Erstellen von Publikationen.

\subsection{Finanzielle Förderung}

Läßt man die Auswirkungen der neuen Medien auf die derzeitigen Bibliotheken Revue passieren, so wird deutlich, daß diese zwar eine größere Effizienz in der Nutzung erbringen, die Aufgaben aber zusätzlich zu den bestehenden erledigt werden müssen. Daher sind zumindest mittelfristig zusätzliche Mittel erforderlich. Sollen die Bibliotheken ihrem bisherigen Auftrag nach Archivierung gleichsam auch für digitale Medien nachkommen, so müssen Fördergelder auch für die Pflege der Systeme und Weiterentwicklung der Standards bereit gestellt werden ${ }^{14}$.

\subsection{Problematiken:}

\section{Copyright}

Die Problematik des Urheberrechts gerade für schnelle und effiziente Ausleih- und Bestelldienste ist bereits vielfach beschrieben worden. Es bleibt zu hoffen, daß sich durch die aus dem BGHUrteil vom Februar 1999 ergebenen Vergütungsverhandlungen auch längerfristig nicht nur die Preise für Kopienlieferungen verändern werden, sondern auch ein ausgewogener Interessenausgleich zwischen Rechtsinhabern und Nutzern entsteht.

\section{Langzeitarchivierung und Sammelauftrag}

Ein recht schwieriges, weil ungelöstes Problem stellt die Archivierung dar. Der Gedanke, Wissen auf einem Medium längerfristig aufzuzeichnen, hat in der Geschichte vermutlich sogleich zu dem Wunsch geführt, möglichst viel Wissen zu sammeln (oder war es doch umgekehrt?). Gleichwohl, die Problematik, ein Medium zu finden, das unabhängig möglichst lange Informationen speichern kann, scheint nicht ohne weiteres lösbar. Natürlich wird zu Recht beklagt, daß beispielsweise CD's je nach Beschichtung vielleicht "nur" eine Lebensdauer von 100 Jahren haben, während Pergament und Papier schon über Jahrhunderte erhalten sind. Dazu passen Meldungen, daß sich gerade durch die Nutzung des Internets der Papierverbrauch durchschnittlich verzehnfacht hat, jedoch wird dabei nicht immer berücksichtigt, daß das heutige "normale" Papier aufgrund seiner Herstellung diese lange Lebensdauer vermutlich auch nicht haben wird, daß es vielmehr durch die darin enthaltene Säure schnell zu einem Zerfall vermutlich noch vor der CD-Lebensdauer kommen wird.

Ein nicht unerheblicher Vorteil des Papiers liegt allerdings darin, daß es innerhalb dieser Zeit auch ohne entsprechende Hard- oder Software seine Informationen preisgibt. Nachteilhaft wirkt sich aus, daß auf Papier nur Wissen in Form von gedruckten Informationen (Schrift, Bilder) enthalten ist, während die CD Audio- und Video-Daten enthalten kann. Wobei hier in der Argumentation ein Punkt erreicht wäre, an dem man sich fragen müßte, ob es wirklich nur um die Problematik des Trägermediums geht. Egal, ob man sich Photographien, Audio- oder Videobänder anschaut, all diese Medien sind oftmals ebenso vergänglich (mit einer geschätzten Lebensdauer von 20 Jahren) und werden, wenn sie archiviert werden sollen, derzeit digitalisiert und damit wieder auf ein anderes Trägermedium "kopiert", allerdings mit der zusätzlichen Problematik, daß zu jeder Information auch eine Wiedergabemöglichkeit (Hard- und/oder Software) bestehen muß, d.h. zu der physischen "Migration" des Datenträgers kommt eine "Konvertierung" des Inhalts, um lesbar zu bleiben. Welches Programm liest heute noch Wordstar-Dateien (Anfang der 90er Jahre sehr gebräuchlich - Prä-WinWord-Area) auf 5 1/4" 360

\footnotetext{
${ }^{13}$ Vgl. die (Denk)Ansätze zu automatisierten Digitalen Bibliotheken, z.B. W.Y. Arms, Automated Digital Libraries. How Effectively Can Computer Be Used for the Skilled Tasks of Professional Libraianship?, in: D-Lib Magazin 6, 2000, Nr. July/August, unter: http://www.dlib.org/dlib/july00/arms/07arms.html.

${ }^{14} \mathrm{Vgl}$. Sietmann, Die Vertreibung (wie Anm. 2), der die Notwendigkeit der Förderung von öffentlichen Einrichtungen betont: (..) das bedeutet in der Bundesrepublik beispielsweise eine Kehrtwendung zur bisher verfolgten Fachinformationspolitik”.
} 
SD-Disketten ein? Es dürfte zudem klar sein, daß jede Konvertierung in ein anderes Format auch gleichzeitig eine Veränderung an der Authentizität des Originals mit sich bringt.

Das Internet stellt uns zudem vor eine weitere Problematik, die es im bisherigen historischen Verlauf in dieser Deutlichkeit überregional noch nicht gegeben hat: die Schnellebigkeit der Information oder anders ausgedrückt die "Flüchtigkeit" der Information. Dies bedeutet kein Werturteil über den Inhalt. Vermutlich ist es gut, daß viele Informationen aktualisiert werden oder eben nicht tradiert werden. Nur, wer möchte diese Entscheidung treffen? Selbst wenn es utopischerweise gelänge, einen Teil der online verfügbaren Informationen zu archivieren, wäre im Prinzip nur ein kleiner Erfolg damit verbunden. Viele Online-Journale zeichnen sich durch schnelles Publizieren und umfangreiche Informationsressourcen mittels Links aus. Gerade diese Verknüpfungen werden aber dazu führen, daß weitere Probleme entstehen. Bei der Fluktuation der Server und Web-Sites werden diese heute noch aktuellen Links morgen bereits "dead"- oder 404-Links sein, da die Information nicht mehr verfügbar ist. Theoretisch müßte eine angepaßte Archivierungsstrategie "Informationscluster" archivieren, die vernetzte Information also gleichsam abbilden, wobei auch hier Veränderungen an der Orginalinformation unausweichlich wären.

Der Auftrag der Bibliotheken, Veröffentlichungen zu sammeln, zu archivieren und zur Verfügung zu halten, sollte auch für digitale Informationen in einem viel stärkeren Maße als bisher wahrgenommen werden. Daß dazu andere Organisationsformen und Zusammenschlüsse nötig sein können, betrifft die Problematik an sich nicht. Es kann dabei auch an Kooperationen und Konsortialverträge mit Verlagen gedacht werden, nur sollten diese Vereinbarungen Nutznießer auf beiden Seiten haben. Auf die Frage, ob durch den von einigen Verlagen forcierten DOI (Digital Object Identifier), der in einem elektronischen Dokument durch Anklicken zu der Fundstelle automatisch verbindet, der Einstieg der Verlage in die Archivierung der wissenschaftlichen Veröffentlichungen eingeläutet würde, antwortete Arnoud de Kemp (Springer) im Januar 2000 ${ }^{15}$ :

\begin{abstract}
“Ja. Wir stehen in interessanten Diskussionen, zum Beispiel mit der Deutschen Bibliothek in Frankfurt. Es ist hocherwünscht, dass die Deutsche Bibliothek die nationale Archivfunktion auch für elektronische Publikationsformen übernimmt. Aber es ist vielleicht nicht notwendig, dass sie dafür sofort alle elektronischen Dokumente einsammelt. Man kann sich vorstellen, dass die Inhalte bei uns liegen, aber dass zwischen den Katalogen der Deutschen Bibliothek und unserem Angebot eine DOIFunktion besteht. Und wenn wir irgendwann ältere Jahrgänge nicht mehr auflegen wollen, weil die nicht mehr gefragt werden, dann können wir sie in Frankfurt bei der Deutschen Bibliothek deponieren. Das einzige, was dann geändert werden mu $\beta$, ist die URL, die hinter dem DOI steht."
\end{abstract}

Die Bibliothek als Archivlager für nicht mehr (nach)gefragte elektronische Dokumente. Sicherlich eine Alternative, jedoch keine, die die eigentlichen oben geschilderten Archivierungsprobleme und die damit verbundenen Kosten löst. Die Frage, die letztlich entscheidend ist, besteht darin, wer zukünftig als Content Provider auftritt. Eine mögliche Folgerung wäre, daß Bibliotheken schon heute beginnen, elektronische Volltextarchive mit professioneller Retrievalfunktionalität größeren Umfangs aufzubauen, und sich nicht allein damit begnügen, Links auf vorhandene Server zu legen oder Meta-Suchmaschinen zu diesen Beständen aufzubauen.

\title{
Eindeutige Identifikation von Publikationen
}

Der zuvor erwähnte Document Object Identifier (DOI) reißt eine weitere Problematik an, mit der zukünftig umgegangen werden muß: Online-Publikationen müssen eindeutig identifizierbar sein. Bereits existierende Numerierungssysteme, wie ISBN oder ISSN, sind im Netz nur bedingt verfüg- bzw. einsetzbar. Bislang benutzt man URL's (Uniform Resource Locator) als

\footnotetext{
${ }^{15}$ Zitiert nach einem interessanten Interview von Richard Sietmann mit Arnoud de Kemp: Eine Zeitschrift (wie Anm. 4).
} 
Bestandsnachweis. Auch diese Vorgehensweise ist problembehaftet, da eine physische Verlagerung des Dokumentes ohne entsprechende automatische Umleitung für den Nutzer sofort zu einem Verweis ins Leere führt. Sofern das Dokument auf dem Server gegen ein anderes Dokument ausgetauscht wird, zeigt der Link über den URL dennoch auf dieses ausgetauschte Dokument, da der URL keinen Hinweis auf den Inhalt sondern nur den Ort des Dokumentes definiert. Zur Lösung dieses Problems wurde der URN (Uniform Resource Name) als flexibles Iditifikationssystem entwickelt, der das Dokument selbst identifiziert und auch bestehende Systeme wie ISBN und ISSN zusätzlich beinhalten kann. Um funktionabel zu sein, muß dazu ein übergeordneter Dienst existieren, der diesen URN's vergibt und die dazu gehörigen URL's in einer Datenbank verwaltet. In Deutschland wird dies vermutlich die Deutsche Bibliothek sein. Die Auflösung des URN in die dazugehörige URL wird zukünftig vermutlich über den Webbrowser ohne Zutun des Nutzers automatisiert passieren.

International haben sich einige Verlage zusammengeschlossen, um den o.a. DOI als praktische Umsetzung des URN über einen Server bei der International DOI-Foundation in Washington zu betreiben. Dieser kommerzielle Dienst ist für die beteiligten Verlage kostenpflichtig.

\section{Digitalisierung (on demand)}

Bei allen Diskussionen über das Ende der "Gutenberg-Galaxis"16 bleibt die Erkenntnis, daß die herkömmliche Publikation noch über einen längeren Zeitraum gegenüber digital erscheinenden Publikationen dominieren wird. In einem Bericht der Kommission für Neue Informations- und Kommunikationstechnologien für wissenschaftliche Bibliotheken beim Bayerischen Staatsministerium für Unterricht, Kultus, Wissenschaft und Kunst (IKB-Kommission) wird geschätzt, daß in nächster Zeit allenfalls $20 \%$ der wissenschaftlichen Neuproduktion digital publiziert werden, oftmals parallel zu einer Druckausgabe ${ }^{17}$. Dies dürfte natürlich von Fachbereich zu Fachbereich mehr oder weniger differieren, zeigt jedoch, daß ein Problem derzeit darin besteht, daß in Bibliotheken einerseits traditionell weitergearbeitet werden muß, während andererseits bereits die Weichen für eine zukünftige Entwicklung gestellt werden sollten. Eine Aufgabe, die bei den bestenfalls stagnierenden Budgets und vorherrschenden Personalstrukturen nicht einfach zu bewältigen sein dürfte.

Abgesehen von den rechtlichen Aspekten, die es zu klären gäbe, ist auch eine retrospektive Digitalisierung kompletter Bibliotheksbestände technisch und finanziell nicht erreichbar. Hierzu existieren über die DFG im Förderbereich "Verteilte Digitale Forschungsbibliothek"

entsprechende Fördermaßnahmen. Darüberhinaus wären Konzepte denkbar, die bei Regelung der Copyright-Problematik eine Digitalisierung on Demand vorsehen. Denkbar ist dieses Verfahren zum Beispiel bereits heute bei der Anforderung von Filmkopien von Handschriftenbeständen oder Wiegendrucken. In den Handschriftenabteilungen liegen bereits heute oftmals

Sicherheitsverfilmungen der Bestände vor, die man auf Anforderung durch den Nutzer statt eines Filmduplikates digitalisieren könnte und letztlich als Kopie in Form einer CD mit den gescannten Bildern z.B. im jpg-Format verschickt. Auch für die Konzeption von Speicherbibliotheken wäre ein angeschlossenes Digitalisierungszentrum, das "on demand" die Bestände digitalisieren würde, eine Überlegung wert.

\section{Wie könnte eine Digitale Bibliothek aussehen?}

Im folgenden soll als Übergang zu dem vorzustellenden Pilotprojekt nur kurz auf einige Charakteristika der Digitalen Bibliothek eingegangen werden, da gerade in der angloamerikanischen Forschung dieses Thema besondere Beachtung gefunden hat. Diann Rusch-Feja hat in einem umfangreichen Aufsatz Hintergründe und Entwicklungen im In- und Ausland

\footnotetext{
${ }^{16}$ Zurückgehend auf: M. McLuhan, Die Gutenberg-Galaxis: Das Ende des Buchzeitalters, Düsseldorf, 1968, Neuauflage Bonn, 1995; Originalausgabe Toronto, 1962.

${ }^{17}$ Bayerisches Staatsministerium für Unterricht, Kultus, Wissenschaft und Kunst. Neue Informations- und Kommunikationstechnologien für wissenschaftliche Bibliotheken. Bericht der IKB-Kommission. München 1997.
} 
aufgezeigt ${ }^{18}$. Sie gibt als Antwort auf das mögliche Aussehen der digitalen Bibliothek u.a.: “(..) der größte Feind der digitalen Bibliothek wird vermutlich die Resistenz gegen Veränderungen sein. Technologischer Fortschritt wird z.B. die vernetzte Umgebung viel stärker einbeziehen, um elektronische Informationsdienstleistungen aller Art über ein Zugangssystem ("one stop shopping”) anzubieten. Dies erfordert jedoch eine relativ hohe Kompetenz im Umgang mit der Umgebung der digitalen Welt, aber auch die gelungene Integration verschiedener Datenbanken und Suchsysteme in eine einheitlich anzusteuernde Oberfläche. Aber wenn die Bibliotheken und Informationseinrichtungen dies zusammen nicht schaffen, wird eine Firma oder eine andere Organisation diese Informationsdienste mit Ihren Preisen anbieten." 19

Das im folgenden beschriebene Pilotprojekt sieht sich gerade durch die Integration mehrerer Datenbanken (OPAC, Z39.50- und SQL-Datenbanken, Volltexte) unter einer einheitlichen Oberfläche mit einem Volltextretrieval im Sinne dieser Digitalen Bibliotheken und möchte als ein modularer Baustein auf dem Weg zu einem umfassenderen digitalen Wissensportal verstanden werden.

\section{Digibib-Hessen -- ein Baustein zur Digitalen Bibliothek}

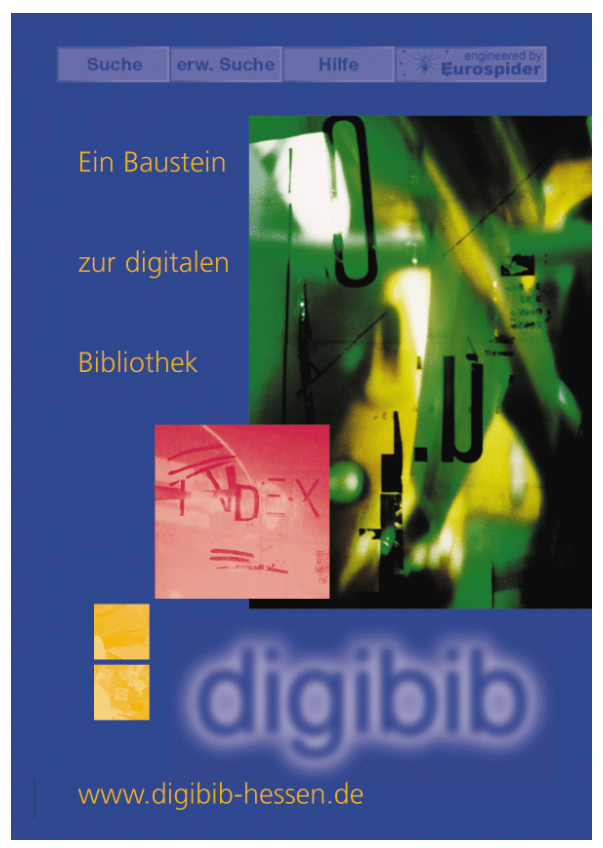

\subsection{Projektverlauf}

Über einen innerhalb der Hessen-Media-Initiative der hessischen Landesregierung 1997 gestellten Antrag der Gesamthochschulbibliothek Kassel sollte eine Lösung für eine einfache und benutzerfreunliche Abfrage (Retrieval) der recht inhomogenen Daten, die mittlerweile in einer wissenschaftlichen Bibliothek über unterschiedlichste Rechnerplattformen und Rechnerzugänge bereitgestellt werden, gefunden werden. Der Projektantrag besagt u.a. zur Zielsetzung des Projektes:
Die projektierte Oberfläche soll für Benutzer einen einfachen Einstieg in eine Vielzahl von unterschiedlichen Datenbanken ermöglichen, die ihm derzeit aufgrund der unterschiedlichen Rechnerplattformen, Retrievalsoftware-Verfahren und fehlenden Verknüpfungen nicht möglich ist.

Es war geplant, eine Kooperation zusammen mit der PICA- Stiftung, Leiden, und der Fa. Eurospider, Zürich, zur Schaffung einer benutzerfreundlichen Oberfläche einzugehen. Diese Zusammenarbeit erschien sinnvoll, da in den Vorbereitungen zu einem hessenweiten Retrodigitalisierungsprojekt Gespräche zwischen allen Beteiligten stattgefunden hatten. Im weiteren Verlauf zeigte sich, daß ein zweigleisiges Verfahren Vorteile bot. PICA hatte mittlerweile ein Projekt namens PiCarta begonnen, in dem es letztlich unter der WebOPACOberfläche multimediale Dokumente verwalten wollte, die zuvor in die PICA-Datenbank katalogisiert und indiziert worden waren. Die erste Beta-Software sollte Ende 1998 zum Test nach Kassel geliefert werden. Mittlerweile existiert in den Niederlanden eine funktionierende PiCarta-Installation, in Deutschland ist die CBS-Version in Göttingen (GBV) installiert.

\footnotetext{
${ }^{18}$ D. Rusch-Feja, Digital Libraries - Informationsform der Zukunft für die Informationsversorgung und Informationsbereitstellung, in B-I-T-Online 2, 3, 4, 1999 und 1,2, 2000, auch unter: http://www.b-i-tonline.de/archiv.

${ }^{19}$ Rusch-Feja, Digital Libraries (wie Anm. 18).
} 


\section{Landesweite Maßnahmen und Dokumentserver}

Im Rahmen des Sonderprogrammes "Wissenschaftliches Schrifttum" des Hessischen Ministeriums für Wissenschaft und Kunst wurden in den Jahren 1997ff. Gelder für landesweite Projekte reserviert. Um die Projektmöglichkeiten auszuloten und entsprechende Vertragsverhandlungen zu führen, wurde in Hessen eine Arbeitsgruppe berufen ${ }^{20}$. In mehreren Arbeitsgruppensitzungen wurden die eingeholten Angebote verschiedener Datenbank-Anbieter diskutiert und anschließend zu einer Gesamtliste zusammengestellt. Generell wurde folgendes festgehalten:

- Die Verträge sollen über alle Fachgebiete reichen, auch wenn dies anhand des unterschiedlichen Entwicklungsstandes bezüglich Online-Medien nicht immer ganz möglich ist.

- Um Bedürfnisse richtig abschätzen zu können und die Möglichkeit eines Weiterbezuges der OnlineMedien durch die jeweilige Bibliothek zu fördern, sollen Eigenbeteiligungen eingeführt werden. Dies erhöht zudem die Anzahl der Angebote nicht unwesentlich.

\section{Beschlossene Maßnahmen}

Laut ministeriellem Erlaß vom 3. November 1998 verteilten sich die zentralen Mittel auf verschiedene Fachgebiete. Gekauft wurden u.a. einige digitale Zeitschriftenarchive, die für diesen Zusammenhang wichtig sind. Diese große Datenmenge kann effektiv nicht mehr mittels Metadaten bearbeitet werden, d.h. herkömmliche bibliothekarische Maßnahmen wie Katalogisierung etc. sind personell und finanziell nicht durchzuführen. Dennoch sind diese Volltexte natürlich für die Benutzerrecherche sehr wichtig, da über einen erzeugten Index sehr qualifizierte Fragen an diese Datenmenge gerichtet werden können. Anschließend kann das gesuchte Dokument, da es digital vorliegt, ohne Zeitverzug am Bildschirm betrachtet werden. Für diese Volltexte mußte also eine Software und ein Dokumentserver zur Erschließung gefunden werden. Da jedoch auch Datenbanken unter Silverplatter und der Bibliotheks-OPAC eingebunden werden sollten, mußte diese Entwicklung eines Dokumentservers konzeptionell erweitert werden.

\section{Entwicklung 1998/99}

Mit der Fa. Eurospider waren vor der Projektbeantragung intensive Gespräche geführt worden, die letztlich darauf hinausliefen, ein Bibliotheksprojekt parallel zu dem damals beginnenden "SwissNet" der deutschsprachigen Schweizer Bibliotheken zu initiieren. Es wurde vereinbart, erste Ergebnisses des "SwissNet" abzuwarten, um dann eine hessische Variante zu implementieren. In 1998 kristalisierte sich immer mehr heraus, daß einerseits die Entwicklungen seitens PICA länger als geplant in Anspruch nehmen würden und andererseits die DVEntwicklung auch bei anderen Projekten in die gleiche Richtung ging wie bei dem vorliegenden Pilotprojekt. Aus diesem Grund wurden gemeinsam mit Herrn Dugall von der StUB Frankfurt/M die vergleichbaren Projekte "Die Digitale Bibliothek NRW" und "IBM Digital Library" in der Ausprägung der Uni Essen besucht und letztlich beschlossen, mit der Firma Eurospider ein eigenes modulares Pilotsystem aufzubauen.

\footnotetext{
${ }^{20}$ Dieser Arbeitsgruppe gehörten an: Herr Dugall (Stadt- und Universitätsbibliothek Frankfurt/M), Frau Klein (FH Wiesbaden), Prof. Dr. Schmiede (Universität Darmstadt), Dr. Steenweg (Gesamthochschulbibliothek Kassel), Prof. Wacker (FH Frankfurt/M). Ab dem 1.1.2000 hat eine Arbeitsstelle unter Leitung von Frau Wiesner (Stadt- und Universitätsbibliothek Frankfurt/M) diese Aufgaben übernommen.
} 


\section{Die Digitale Bibliothek NRW (http://www.digibib-nrw.de)}

Über eine großzügige finanzielle Förderung war es im Bundesland Nordrhein-Westfalen unter Regie der UB Bielefeld möglich, eine Digitale Bibliothek aufzubauen und über

Konsortialverträge entsprechende Zugriffe auf Daten zu erwerben.

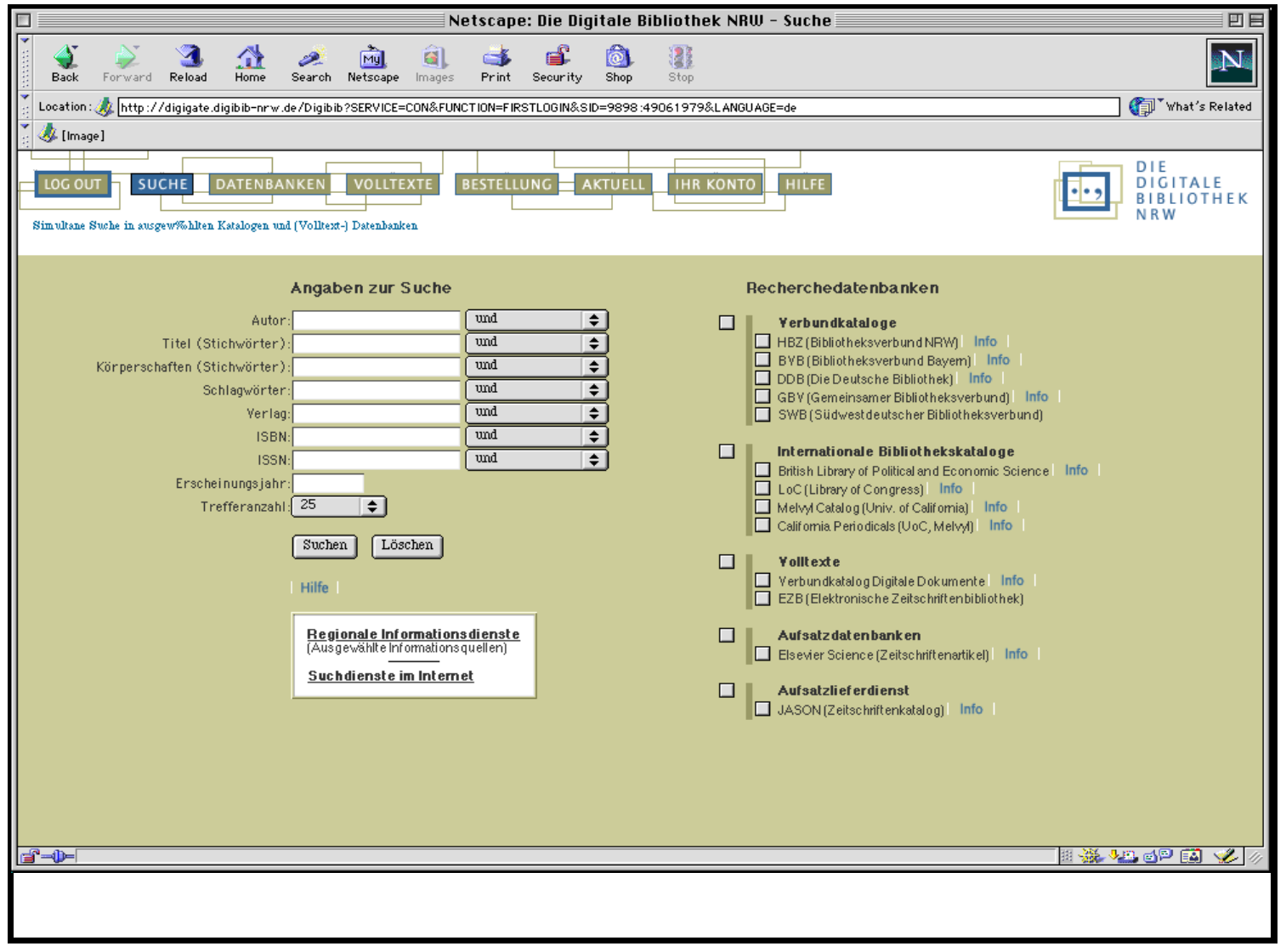

Die Digitale Bibliothek NRW entstand in Zusammenarbeit mit der Fa. IHS, München. Dieses Projekt stellt derzeit eine je nach beteiligter Bibliothekssicht unterschiedliche Meta-

Suchmaschine zu Anbietern und Betreibern digitaler Datenbestände dar. Die einzelnen Spider liefern über normierte Schnittstellen (derzeit Z39.50, SQL und HTTP) Ergebnisse aus den jeweils befragten Retrievalsystemen. Diese Ergebnisse werden in einer Ergebnisliste dargeboten, allerdings nicht vereint sondern hintereinander je nach zeitlichem Eintreffen. Über entsprechende URL's kann der Benutzer anschließend weitere Informationen zu diesen digitalen Dokumenten auswählen. Zum System, das über einen zentralen Server in Bielefeld und einen Mirror-Server beim HBZ in Köln verfügt, gehört weiter ein Authentifizierungssystem, ein Administrationstool, ein Abrechnungsmodul sowie eine Collectdatenbank auf Basis einer Datenbank der Fa. IHS. Dieses System ist derzeit das am weitesten entwickelte System in Deutschland, bedingt jedoch durch die Auslegung als Metasuchmaschine keine Bearbeitung von Volltexten (PDF etc.) oder Multimedia-Formaten und gestattet zudem nur einen statischen Zugriff auf kostenpflichtige Dokumente, beinhaltet also im Gegensatz zu dynamischen Links die Problematik, daß diese Links kopiert werden können.

\section{Projekt MilESS - IBM Digital Library der Uni Essen (http://miless.uni-essen.de/)}

Das Projekt MilESS, gegründet als Projekt der Bibliothek und des Hochschulrechenzentrums in Essen mit den Fachbereichen Physik und Linguistik, sollte die speziellen Nöte der betreffenden Fachbereiche mit Multimedia-Daten beheben. Auf Basis der DB2-Digital Library von IBM 
wurde in intensiver Arbeit eine Arbeitsumgebung in JAVA zusammengestellt, die es ermöglicht, die Digital Library parallel zum OPAC zu befragen. Beide Suchergebnisse werden zwangsläufig derzeit getrennt ausgewiesen Es wird in der Digital Library ebenso möglich sein, digitale Dokumente gemäß Dublin-Core-Richtlinien abzugeben.

Um Dokumente in dieser letztlich sehr komfortablen Umgebung recherchieren zu können, muß jedoch jedes Dokument in die Digital Library katalogisiert werden. Dieser Arbeitsaufwand und die Umarbeitung der von IBM zur Verfügung gestellten Tools innerhalb der Digital Library hin zu einer landesweiten Lösung sind nicht unerheblich.

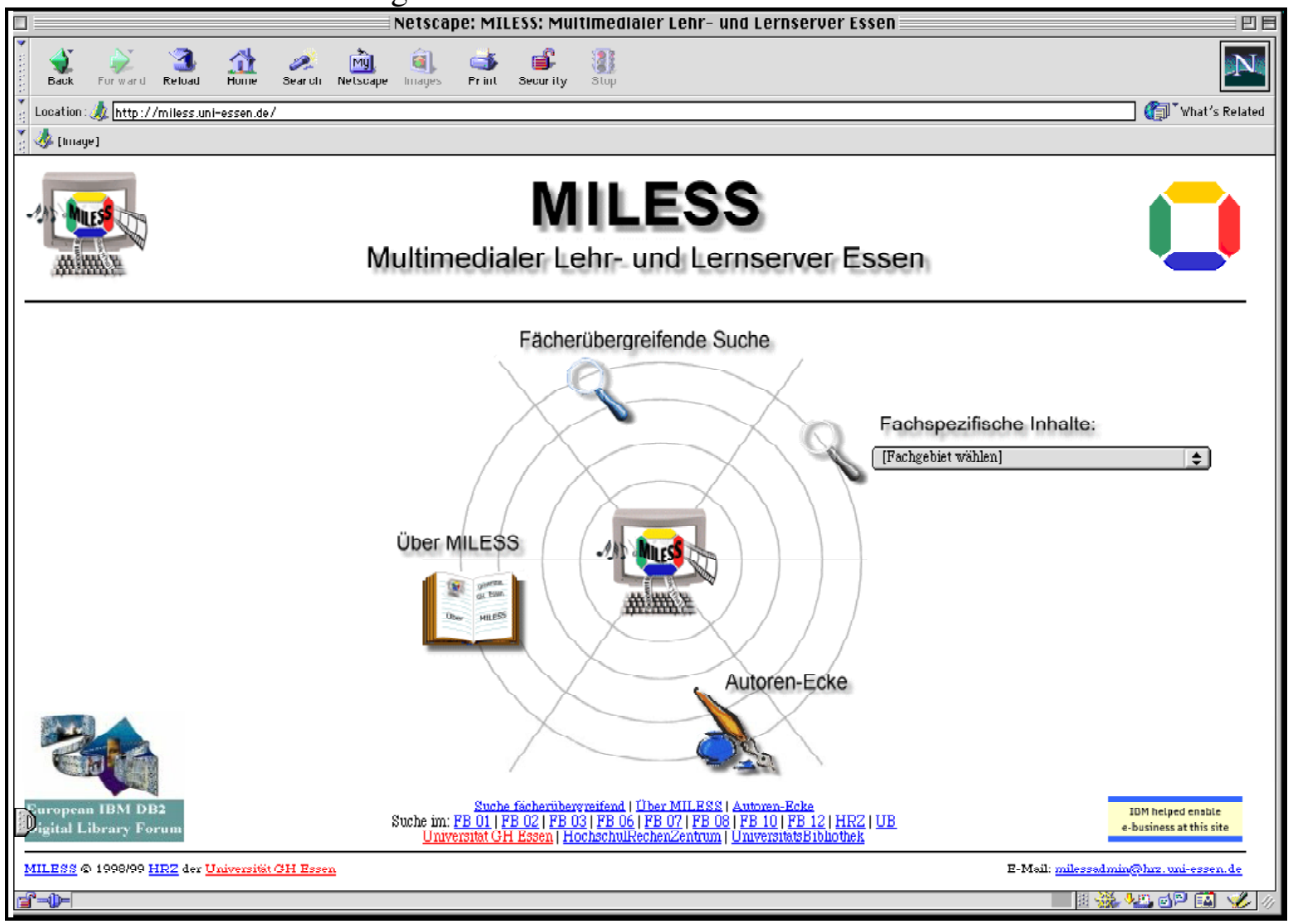

\section{PiCarta (http://picarta.pica.nl)}

Derzeit existiert eine funktionierende holländische Version und eine CBS-Version in Göttingen. Bei der LBS-Version fehlte bislang die notwendige Anbindung an das Ausleihsystem.

Jedes zu recherchierende Element muß in das PiCarta-System katalogisiert resp. verknüpft werden. Es sind weiterhin derzeit nur die Datenbanken zu integrieren, die von Pica unterstützt werden. PiCarta stellt eine elegante Verknüpfung eines Katalogsystems mit Volltexten dar. Eingangssystem ist systembedingt die Katalog-Sicht. Da keine Volltextindizierung derzeit vorgesehen ist, kann entsprechend nur in den Metadaten gesucht werden, die zum Metadatensatz gehörigen Volltexte sind verlinkt aber nicht indiziert. 


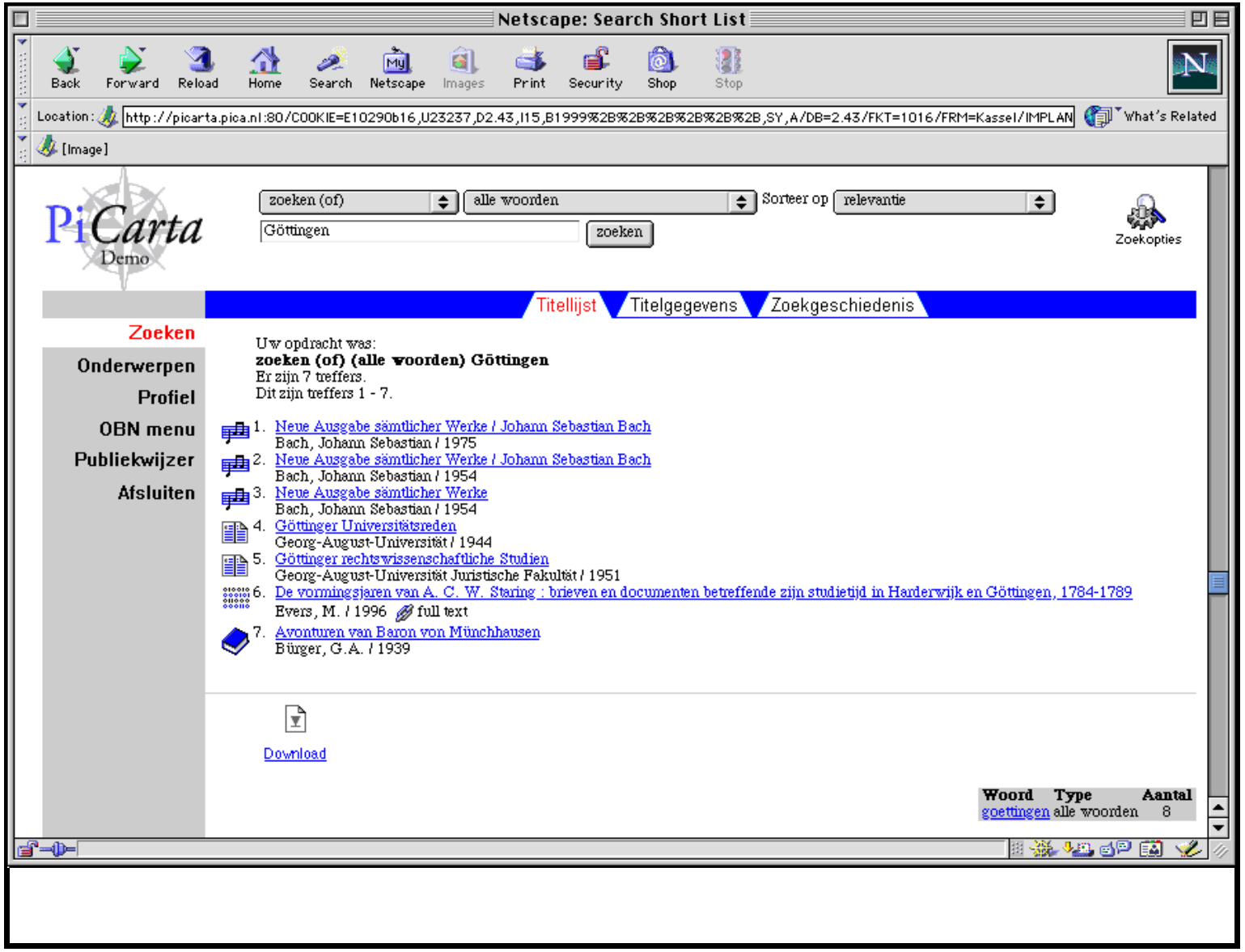

\subsection{Das Pilotsystem (http://www.digibib-hessen.de)}

digitale

bibliathek

hessen

\begin{tabular}{|l|l|}
\hline \multicolumn{2}{|l|}{ Login-Information } \\
\hline Profil: & hessen \\
\hline Sprache: & de,pdf \\
\hline Session: & \\
\hline
\end{tabular}

Session:

Um Ihre Rechte zu erveitem vählen Sie bitte Logi

Dies ist eine Testinstallation. Es werden Cookies (wird bei Beenden des Browsers gelöscht) und JavaScript benötigt ! 
Betrachtet man die in der Projektbeantragung genannten Ziele, so wird man feststellen, daß die Intention 1997 schon deutlich in die Richtung gingen, die heute allgemein als "Digitale Bibliothek" beschrieben werden. Vergleicht man die unterschiedlichen Ansätze und Projekte, wie z.B. "Global-Info", "Digitale Bibliothek NRW", "IBM Digital Library" so unterscheiden sich die Projekte nicht nur in ihrer Hard- und Softwareausgestaltung. Insbesondere der Denkansatz ist mindestens ebenso unterschiedlich. Ein wesentliches Kriterium liegt beispielsweise darin begründet, wie die Projekte mit Volltexten umgehen.

\section{Volltextrecherche}

Zwar versteht sich auch das vorliegende Projekt deutlich als dienstleistender Informationsvermittler, jedoch liegt der Schwerpunkt nicht auf dem reinen Durchreichen von Informationen als umfassende Informations-Linksammlung, sondern es soll der Ansatz verfolgt werden, die dargebotenen Informationen möglichst auch sofort als Volltext darbieten zu können. Für eine solche Vorgehensweise sprechen mehrere Gründe:

- Es kann nicht immer gewährleistet werden, daß der externe Informationsanbieter das Angebot langfristig aufrechterhalten kann. Kommerzielle Strukturen und Veränderungen, insbesondere im Verlagswesen, greifen sehr viel schneller und nachhaltiger als bei öffentlichen Institutionen.

- Externe Informationsanbieter werden fast immer auf die eigene proprietäre Such- und Indexmaschine verweisen, so daß stetig Anpassungen, auch bei sog. Standards wie Z39.50, nötig sind.

- Die zu benutzenden Indexdatenbanken von externen Anbietern sind möglicherweise nach anderen Kriterien aufgebaut. Da die Daten nicht von der eigenen Indexmaschine bearbeitet worden sind, gelten für diese Daten andere Strukturinformationen als bei den eigenen Indexdaten. Es ergeben sich somit noch stärker inhomogene Suchergebnisse.

- Einmal erworbene Daten (Landeslizenz) müssen zukünftig nicht mehr erworben werden.

- Es entspricht dem bisherigen Erwerbungsvorgang, z.B. einen erworbenen Zeitschriftenjahrgang zur Benutzung ins Regal zu stellen. Sofern das elektronische Pendant auf einen Server gelegt wird, hat dies eine Entsprechung. Kauft man nur das Zugriffsrecht für einen definierten Zeitraum, so ist der Zeitschriftenjahrgang nach Ablauf der vereinbarten Zugriffszeit in der Regel nicht mehr für den Benutzer vorhanden.

Daher wurde in Hessen bei den Konsortialverhandlungen darauf geachtet, nicht nur ein Zugriffsrecht auf bestimmte elektronische Medien für einen bestimmten Zeitraum, sondern gleichzeitig auch sog. "Back-Files" der entsprechenden Medien zu erwerben, damit auch nach dem definierten Zugriffszeitraum ein unproblematischer Zugriff möglich ist. Ausgehend von diesem Dokumentserver-Prinzip ist folglich ein komfortabler Umgang mit PDF-Dateien, dem mittlerweile durchgängig anerkannten Beschreibformat für elektronisch publizierte Texte, nötig. 


\section{Hardware der DigiBib-Hessen}

Als Hardware ist derzeit eine SUN 450 Enterprise mit zwei Prozessoren und zwei externen RAIDs sowie ein Linux-Server im Einsatz.

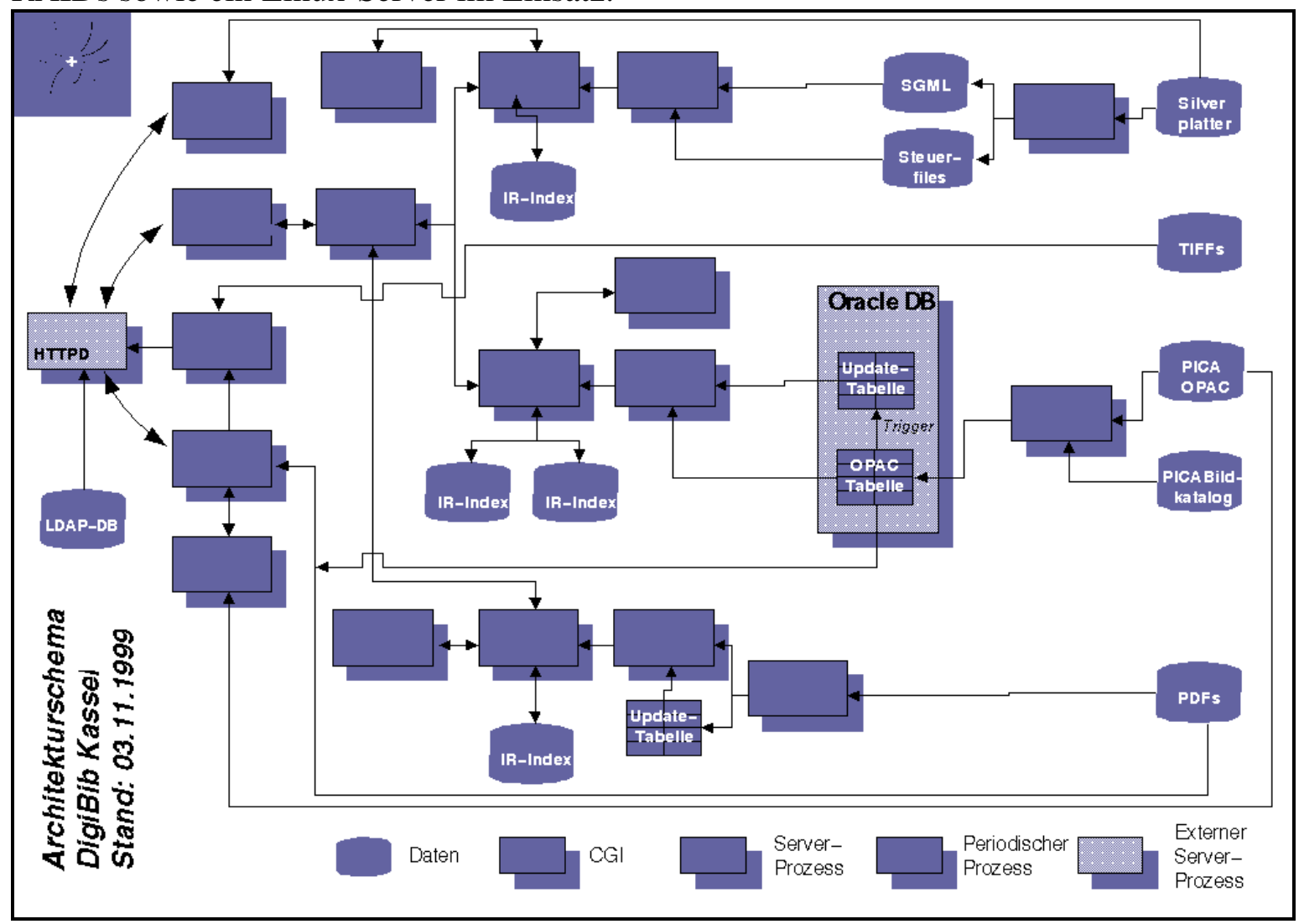

\section{Software der DigiBib-Hessen}

Aufbauend auf einen Apache-HTTP-Server wurden neben der freiverfügbaren Software LDAP und der Forum-Software Komponenten der Fa. Eurospider benutzt. Als Such- und IndexSoftware kommt der Rotondo-Spider der Fa. Eurospider zum Einsatz.

\section{Backup - ADSM}

Das Sicherung der Daten erfolgt in Kooperation mit dem Hochschulrechenzentrum über ADSM.

\section{Inhalt der DigiBib-Hessen}

Derzeit sind im Testsystem folgende Systeme integriert bzw. Schnittstellen geschaffen worden für: 


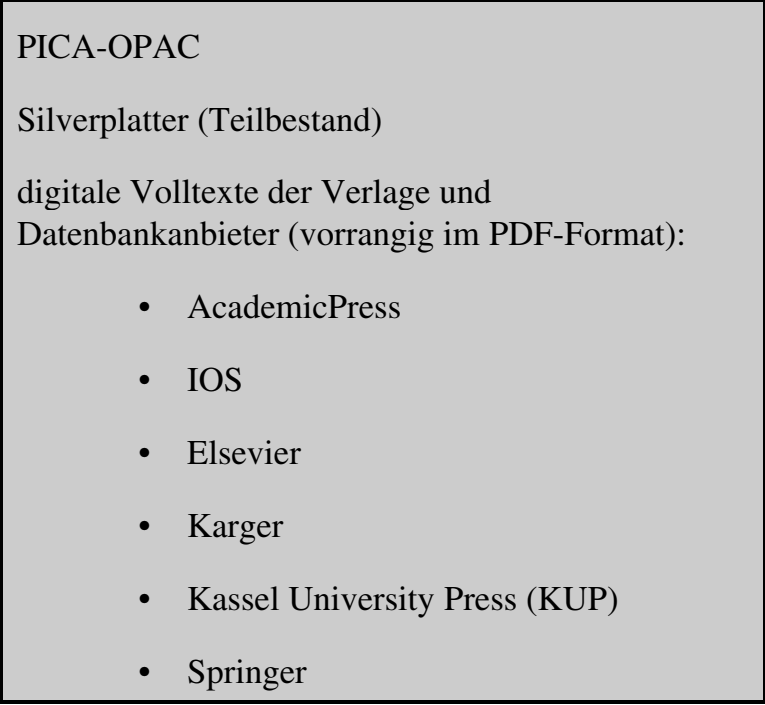

In Zahlen beträgt der derzeitige Stand (1.7.2000) der Online-Archive für Zeitschriften, Monographien und Metadaten:

\begin{tabular}{|ll|}
\hline OPAC KS & 527.000 Datensätze (Rest von \\
Silverplatter & 3 Datenbanken \\
Springer & 7 Zeitschriften (1. Lfg. von 84) \\
KUP & 21 Monographien (komplett) \\
Karger & 78 Zeitschriften (komplett) \\
IOS & 1 Zeitschrift (komplett) \\
AP & Testdaten \\
\hline
\end{tabular}

Dennoch summieren sich allein die digital vorliegenden obigen 86 Zeitschriften mit all den vorhandenen Jahrgängen und Ausgaben auf 13.500 PDF-Dateien. Rechnet man diese Relation hoch auf die im Laufe des Jahre 2000 zu erwartende Menge von derzeit 1100 Zeitschriften, so bedeutet dies eine Menge von 173.000 PDF-Dateien, die es zu verwalten, zu recherchieren und komfortabel anzubieten gilt. Da von jedem Datensatz unterschiedliche Indizierungsmerkmale im System aufgenommen werden, sind die daraus resultierenden Datenmengen erheblich höher anzusetzen. Je nach Spezifikation der jerweiligen Datenquelle fällt die Relation DokumentIndizierungsmerkmal sehr unterschiedlich aus. Der derzeitige Bestand (1.7.2000) ist in folgender Tabelle dargestellt: 


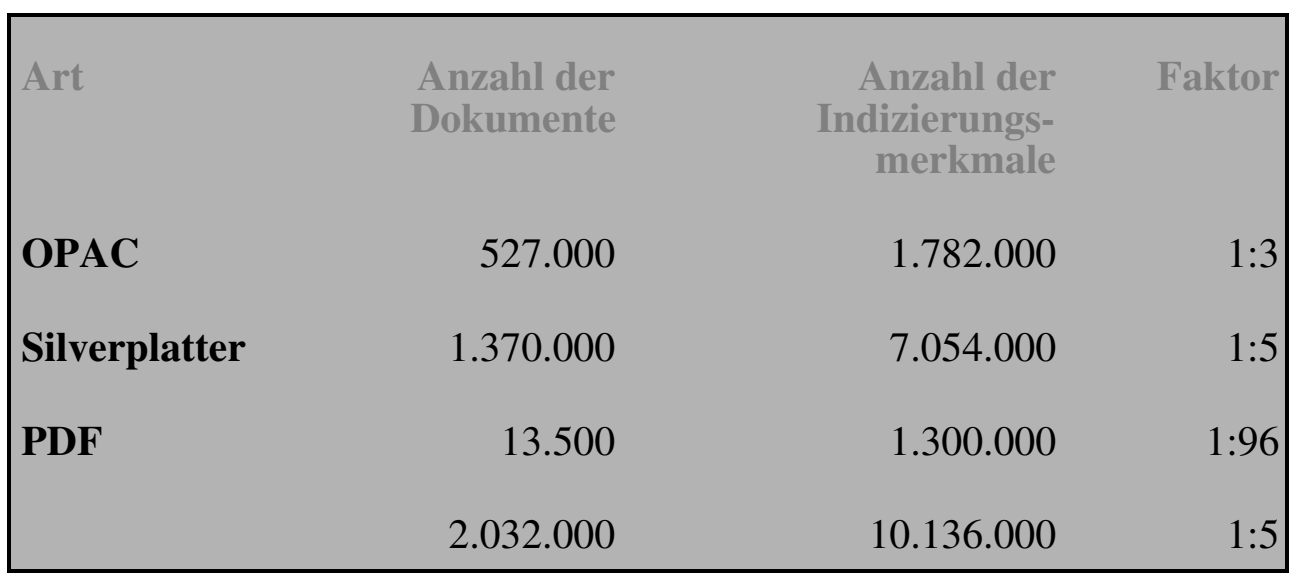

Rechnet man die zu erwartenden PDF-Datenbestände wieder unter diesen Ergebnissen hoch, so sind von 173.000 PDF-Dateien ungefähr 17.300.000 Indizierungsmerkmale zu erwarten.

\section{Funktionsweise DigiBib-Hessen}

Die DigiBib-Hessen ist unter der verbundeigenen Domain erreichbar:

http://www.digibib-hessen.de

Die Notation richtet sich nach der von Nordrhein-Westfalen vorgegebenen Struktur:

$$
\text { "digibib-bundesland.de". }
$$

Wie aus der über alle Webseiten stets sicht- und benutzbaren Navigationsleiste zu erkennen ist, teilt sich die "DigiBib-Hessen" in folgende Bereiche:

- Eingang, Identifizierung, Rechteerwerb auf den Zugriff von elektronischen Dokumenten

\section{Login}

- Blättern in Beständen, Suche in Volltexten, Suche in speziellen Feldern (Autor, Titel - soweit vorhanden), Upload von elektronischen Dokumenten, z.B. Dissertationen.

\section{Blättern}

- Kommunikations- und Informationsbereich mit Forum zum direkten Austausch von Nachrichten, Anzeige von Neuigkeiten zum System sowie einer Linksammlung zu Themen im Umfeld der digitalen Bibliothek.

\section{Forum News Links}

- Letztlich der Bereich der Hilfe, in dem Hilfetexte die Besonderheiten des Systems erläutern sollen.

\section{Impressum Hilfe}

Der zentrale Bereich der DigiBib-Hessen liegt bei den Punkten: Blättern-Suche-Upload. Heutige Benutzer werden mit unterschiedlichen Fragestellungen an eine digitale Bibliothek herantreten. Es wird zum einen Benutzer geben, die genau wissen, welchen Aufsatz sie möglichst schnell lesen möchten. Für diese Benutzer stellt die "DigiBib-Hessen" eine Baumstruktur zur Verfügung, mittels derer sich die Benutzer sehr schnell zu dem gesuchten Aufsatz einer Zeitschrift navigieren können. 


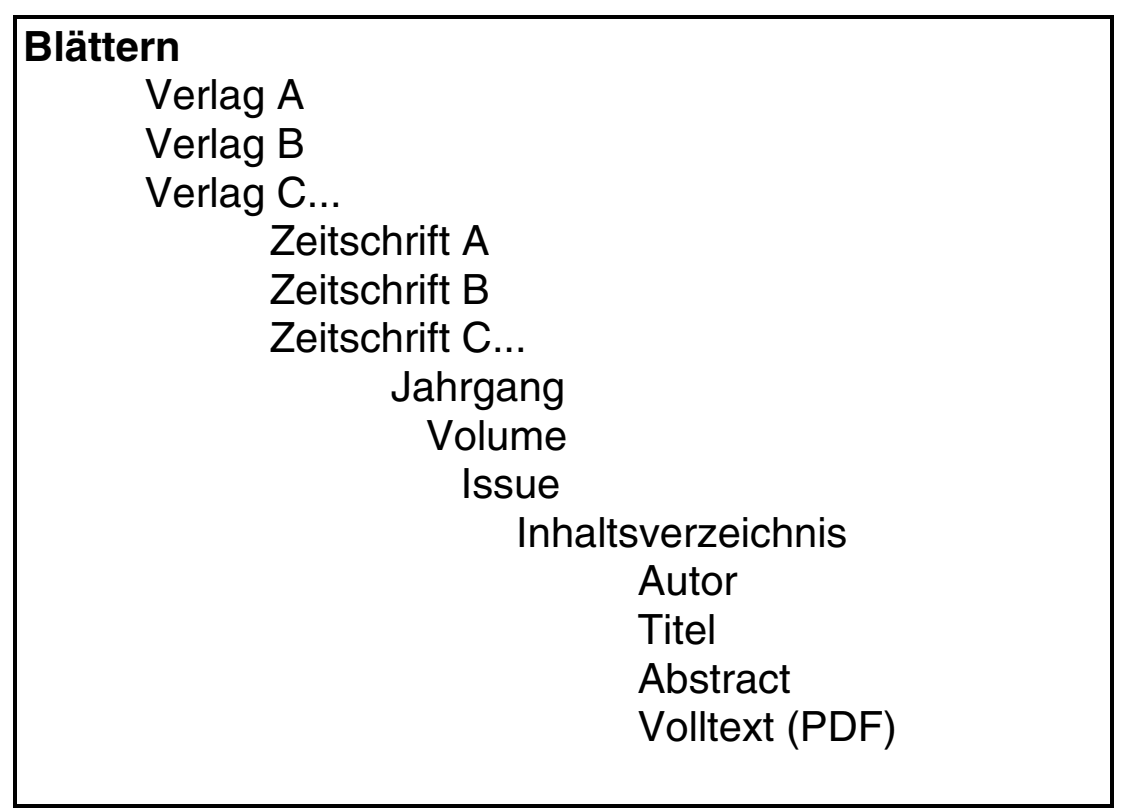

Da die gelieferten Datenbestände der Verlage sehr inhomogen sind, lassen sich nicht für alle Verlage derartige Baumstrukturen aufbauen, jedoch ist erfreulicherweise zu vermerken, daß der Trend deutlich zur Lieferung umfangreicher Informationen geht, die oftmals bereits in SGMLStrukturen angeboten werden.

Eine andere denkbare Benutzersicht bei der digitalen Bibliothek wird die Suche oder anders benannt das Retrieval sein. Dabei ist an Vorgehensweisen eine eher als offen zu bezeichnende von einer spezifizierten Suchstrategie, bei der gezielt nach einen in Feldern kategorisierten Begriff gesucht wird (z.B. Autorensuche), zu unterscheiden. Dem zufolge differenziert die "DigiBib-Hessen" zwischen Volltext- und spezieller Suche mit Kategorien (z.B. Autor, Titel). Die spezielle Suche kann naturgemäß nur bei den Datenbeständen angewandt werden, die über entsprechende Felder verfügen. Derzeit ist das Testsystem noch derart ausgelegt, daß spezielle Suchen pro Datenbestand angeboten werden. Es ist jedoch daran gedacht, diese verschiedenen speziellen Suchen zu einem einzigen Suchformular zusammenzufassen.

Neben der Suche von Informationen dürfte heutzutage ein wesentlicher Bestandteil einer digitalen Bibliothek auch in der Abgabe elektronischer Dokumente liegen. Dem ist in der "DigiBib-Hessen" unter dem Punkt "Upload" Rechnung getragen worden. Sofern ein Benutzer ein digitales Dokument abgeben möchte, erhält er unter dem Punkt Dokument die Möglichkeit, eine entsprechenden Abgabe zu machen. Sofern er bereits unter seiner LDAP-Kennung (Identifizierung über "Login") andere Dokumente abgegeben hatte, würden diese ebenfalls unter Dokumente angezeigt. 


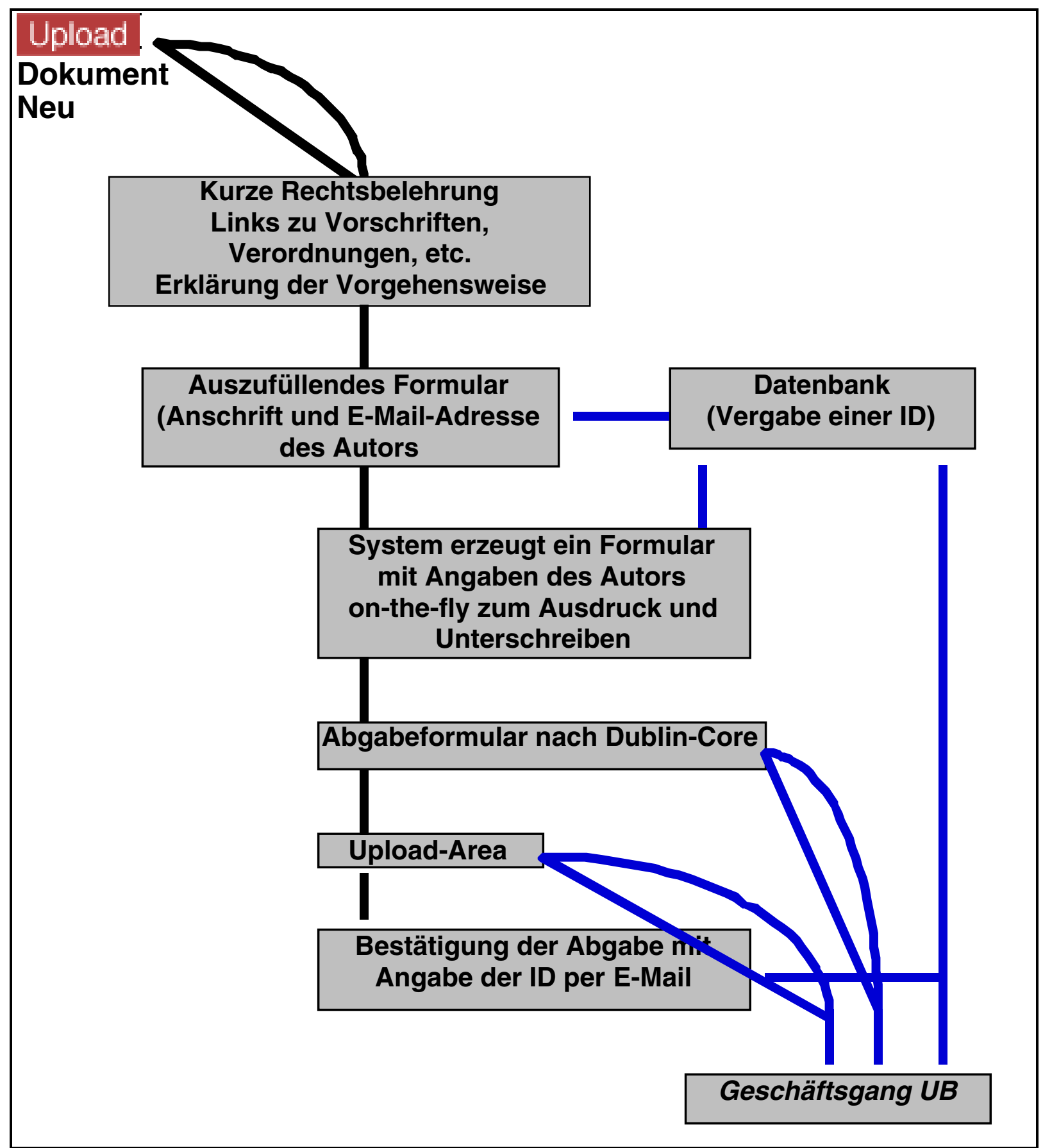

Im Hauptframe des Browsers würde eine Kurzfassung der Rechtsbestimmungen mit entsprechenden Links zu den ausführlichen Vorschriften und Verordnungen sowie eine Erklärung der Vorgehensweise folgen. Sofern diese Rechtsbestimmungen akzeptiert werden, erscheint ein Formular, in dem der Autor Anschrift und E-Mail-Adresse einträgt, die in einer Datenbank gespeichert werden. Aus diesen Datenbankangaben, die um eine Identifikationsnummer ergänzt werden, generiert das System on-the-fly ein Formular mit den zuvor angegebenen Autorangaben. Dieses Formular kann sich der Autor ausdrucken; es muß anschließend als unterschriebene Einwilligungserklärung der jeweiligen Bibliothek geschickt werden. Im Hauptframe erscheint anschließend ein ausführliches HTML-Formular nach Dublin Core (gemäß DDB-Richtlinien), das nach dem Ausfüllen (mit Konsistenzprüfungen seitens des Systems) die Upload-Area freigibt. Nach erfolgter Abgabe erhält der Autor eine Bestätigung der Abgabe per E-Mail unter 
Angabe der o.a. ID-Nummer. Die jeweilige Weiterbearbeitung nach dieser Dokumentabgabe innerhalb der jeweiligen Bibliothek muß über Geschäftsgänge geregelt werden. Unter dem Punkt "Sicherheit" kann der Autor festlegen, inwieweit sein Dokument öffentlich zugänglich sein soll.

\section{Login mittels LDAP (Light-weight Directory Access Protocol)}

Die Anmeldeprozedur erfolgt über eine LDAP-Implementation, mit der sichergestellt werden kann:

- Im Gegensatz zum bislang häufig verwandten IP-Checking kann beim LDAP ein Benutzer stets die Rechte unabhängig von der Rechner-IP erhalten, die ihm zustehen; unabhängig, an welchem Rechner er sich befindet.

- Die Rechteverwaltung kann bis auf einzelne Texte heruntergebrochen werden, so daß z.B. einzelne Texte nur für bestimmte Personen recherchierbar sind.

Derzeit existiert für dieses System eine LDAP-Installation mit Benutzerkennungen des PICALokalsystems (LBS) Kassel (ca. 40.000 Einträge). Sofern andere Hochschulbibliotheken sich diesem System anschließen, können weitere LDAP-Server dezentral an den jeweiligen Hochschulstandorten in dieses System integriert werden, so daß die Verwaltung der Nutzerdaten stets im jeweiligen System verbleibt.

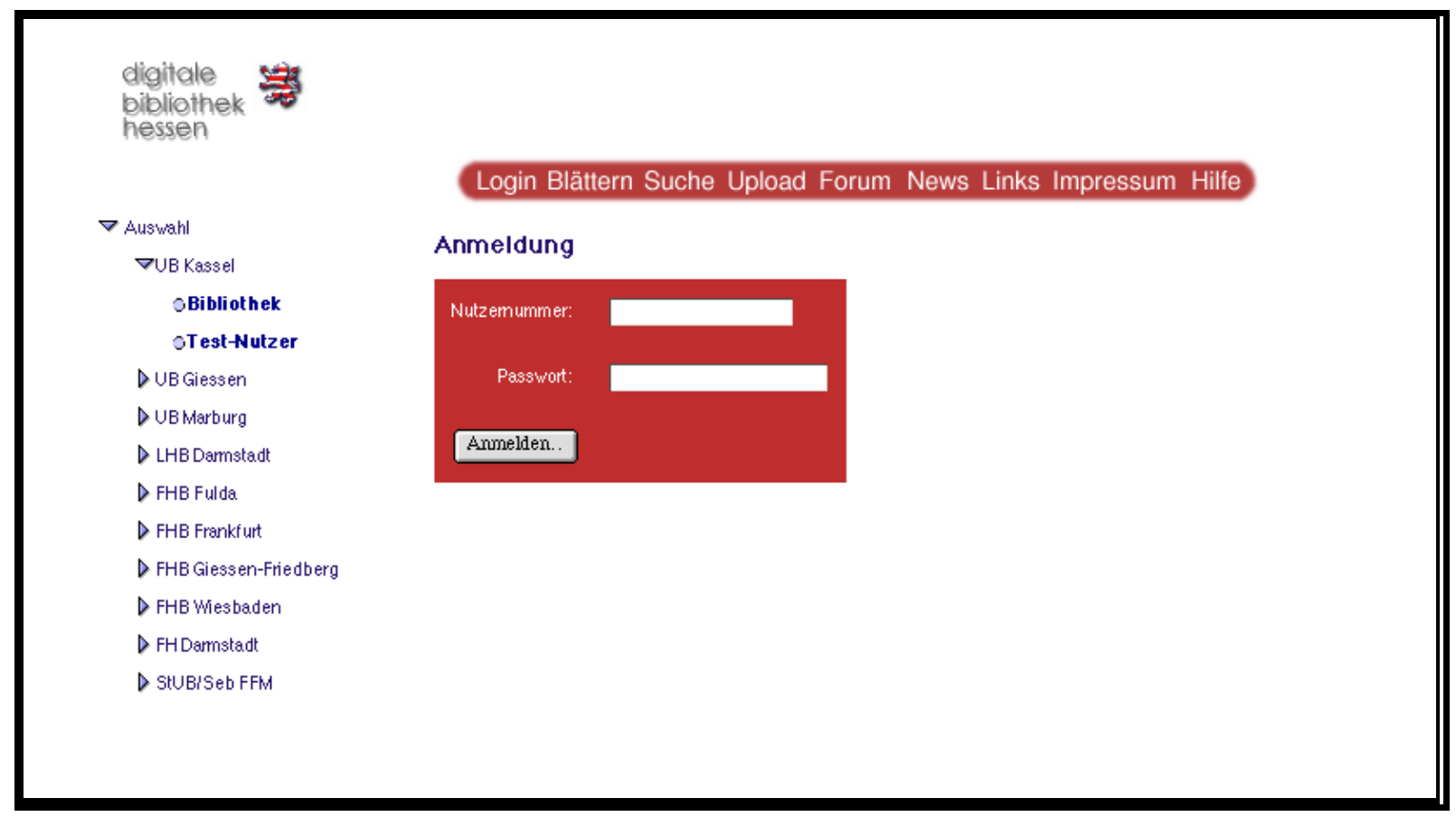

Ein solcher LDAP-Eintrag sieht wie folgt aus:

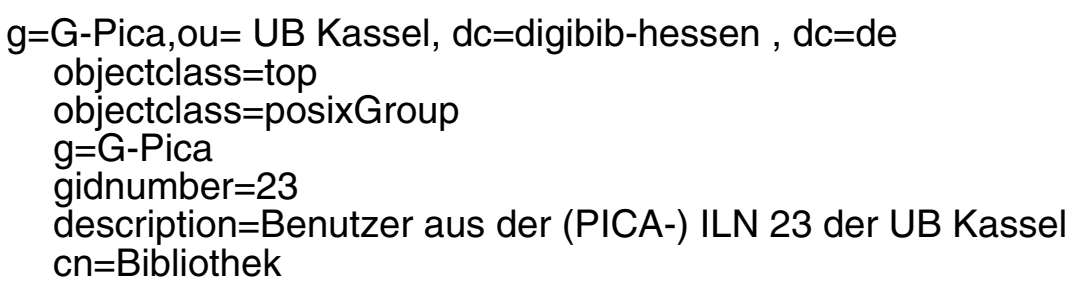




\section{Navigationsleiste}

Über die mittig angebrachte Navigationsleiste sind die wichtigsten Funktionen des Systems stets erreichbar.

\section{Login Blättern Suche Upload Forum News Links Impressum Hilfe}

\section{Blättern}

Über die Funktion "Blättern" kann der Nutzer einen bestimmten Volltext, z.B. einen bestimmten Jahrgang einer elektronisch vorliegenden Zeitschrift über eine Baumstruktur anwählen und anschließend über das Acrobat-PlugIn sofort betrachten, ohne die Suchfunktion nutzen zu müssen.

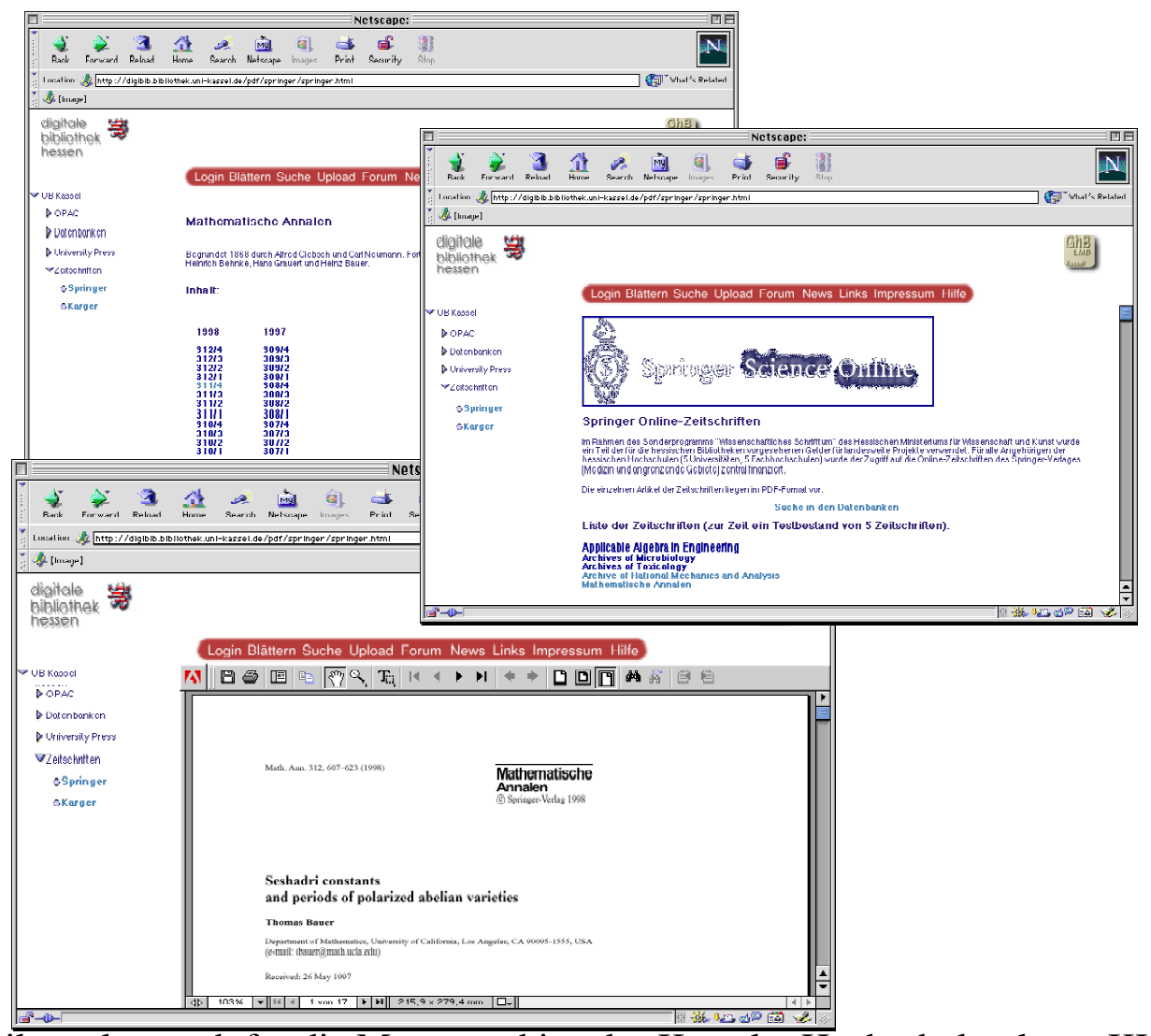

Dies gilt analog auch für die Monographien des Kasseler Hochschulverlages KUP, dessen elektronische Dokumente über diese Baumstruktur komfortabel betrachtet werden können. Diese Dokumente sind derart gesichert, daß sie zwar am Bildschirm betrachtet, aber nicht gedruckt werden können. Sofern dies gewünscht wird, muß über den Verlag ein Entgeld bezahlt werden.

\section{Suche}

Besondere Bedeutung kommt dem eigentlichen Suchsystem, das gleichzeitig auch die Benutzerschnittstelle darstellt, zu. Der Benutzer möchte ein einfach zu bedienendes, möglichst umfassendes, aber dennoch auf seine Bedürfnisse ausgelegtes System vorfinden.

Auf dieses Retrieval wurde daher bei den Überlegungen zur DigiBib-Hessen besonderes Gewicht gelegt. Damit der Benutzer bei einer Anfrage die für ihn wichtigen Antworten möglichst weit oben in einer Rangliste erhalten kann, müssen im Hintergrund die Fundstellen gewichtet werden, d.h. nach ihrer Relevanz absteigend rangiert werden. Von dem System wird für jedes gefundene Dokument ein Relevanzwert geschätzt, der die Wahrscheinlichkeit ausdrückt, mit der dieses Dokument bezogen auf die Anfrage relevant ist, und anschließend innerhalb einer Rangliste 
präsentiert. Hierbei werden die Dokumente zunächst für einen Index mit verschiedenen informationslinguistischen Verfahren behandelt:

\section{- Buchstabenumwandlung}

Hierbei werden Worte "normiert", z.B. durch Umwandlung bzw. Umschreibung von Sonderzeichen und Sonderformen, z.B. vom 8-bit-ASCII zu 7-bit-ASCII oder Groß- zu Kleinschreibung. (größer $->$ groesser)

- Wortextraktion

- Stoppwortelimination Ausschluß von Artikeln, Adverbien, Füllwörtern, etc. (in der heutigen Gesellschaft -> heutigen Gesellschaft)

- Wortzerlegung, Wortstammanalyse (stemming) Ausschluß von Prä- oder Suffixen. In Englischen ist diese Verfahrensweise relativ einfach durchführbar, im Deutschen bedient man sich dazu meist der Hilfe von Wörterbüchern. (Informationsgesellschaft $->$ information gesellschaft)

- Wortnormalisierung (gestellt ->stellen $->$ stell)

Auf den so gewonnenen Datenbestand werden bei einer Anfrage Rangierprinzipien angewandt. Bei den bekannten Internet-Suchmaschinen, wie AltaVista, Excite, Infoseek, etc. lassen sich nicht immer klare Aussagen machen, nach welchen Rangierprinzipien Ergebnisse präsentiert werden. Im Prinzip lassen sich mindestens sechs Verfahren benennen, nach denen heute in der Praxis Suchergebnisse gewichtet werden:

- $\quad$ Anzahl der Suchbegriffe im Dokument

- $\quad$ Termhäufigkeit

- $\quad$ Dokumentenhäufigkeit

- $\quad$ Dokumentenlänge

- $\quad$ Proximity

- $\quad$ Position des Suchbegriffes im Text

Die drei ersten Rangierprinzipien dürften in fast allen professionellen Suchsystemen enthalten sein, auch der letzte Punkt bezüglich der Position findet häufig dadurch Anwendung, daß die Überschriften separat indiziert und gewichtet werden. In der der "DigiBib-Hessen" zugrundeliegenden Eurospider-Indizierungssoftware werden alle beschriebenen Rangierverfahren angewandt. Bei der Suche nach Informationen kann eine weitere Methodik schnell von Nutzen sein. Oftmals kann der Suchende sehr schnell verifizieren, welche Dokumente in der Ergebnisliste seiner Erwartung am besten entsprechen. Bei der Relevanzrückkopplung wird mit den indizierten Begriffen der/des verifizierten Dokumente(s) eine neue Anfrage generiert, die aus Suchbegriffen besteht, die in den/dem relevanten Dokument(en) häufig vorkommen. Damit werden weitere relevante Dokumente gefunden, auch wenn sie keine Suchbegriffe der ersten Anfrage enthalten.

\section{Suchanfrage}

Um Suchanfragen an das System zu stellen, genügt es, einen beliebigen Suchtext in das Eingabefeld der Suchmaske einzugeben, wobei dies unproblematisch auch eine ausformulierte Frage sein kann. Das System analysiert die Sprache der Anfrage, um weiterführende Rangierprinzipien durchführen zu können (Problem z.B.: unterschiedlicher Ausschluß von Präoder Suffixen im Deutschen und im Englischen). 
Bitte Suchbegriffe eingeben (Deutsch, Französisch, Itadienisch, Englisch)
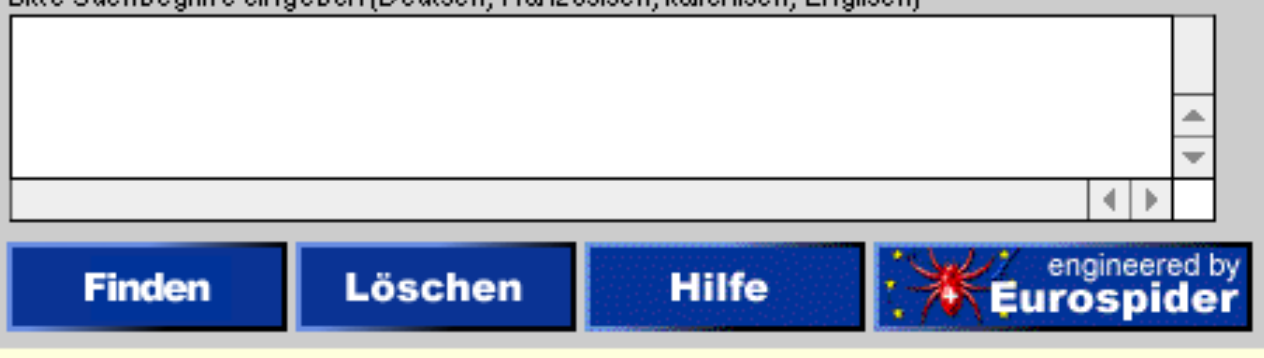

[Subkollectionen und Einschrönkungen anzeigen] [URL hinzufiugen]

Uneingeschränkte Suche in allen Dokumenten.

Der Suchtext kann frei eingegeben werden, es gibt keine Einschränkung hinsichtlich der Formulierung. Je mehr Suchbegriffe eingegeben werden, umso besser wird das Suchresultat ausfallen.

\section{Finden}

Nach Eingabe des Suchtextes muß der "Finden"-Knopf geklickt werden. Die Anfrage kann in jeder beliebigen Sprache gestellt werden, es ist jedoch empfehlenswert, entweder Deutsch, Englisch, Französisch oder Italienisch zu verwenden. Bei diesen Sprachen kann das System eine linguistische Analyse der Anfrage vornehmen, was das Suchresultat erheblich verbessert. Das Eurospider-System extrahiert automatisch die wichtigen Suchbegriffe in der Anfrage und eliminiert die sog. Stop-Worte, die keine Bedeutung für die Suche tragen. Das probabilistische Suchsystem von Eurospider ignoriert logische Boole'sche Operatoren wie 'UND (bzw. 'AND'), 'ODER' (bzw. 'OR') etc., da wissenschaftliche Untersuchungen gezeigt haben, daß probabilistische Suchsysteme Boole'schen Systemen in Effizienz und Effektivität überlegen sind. Eine Suche erstreckt sich normalerweise auf den gesamten Dokumentenbestand. Die Suche kann jedoch auf eine oder mehrere Subkollektionen eingeschränkt werden

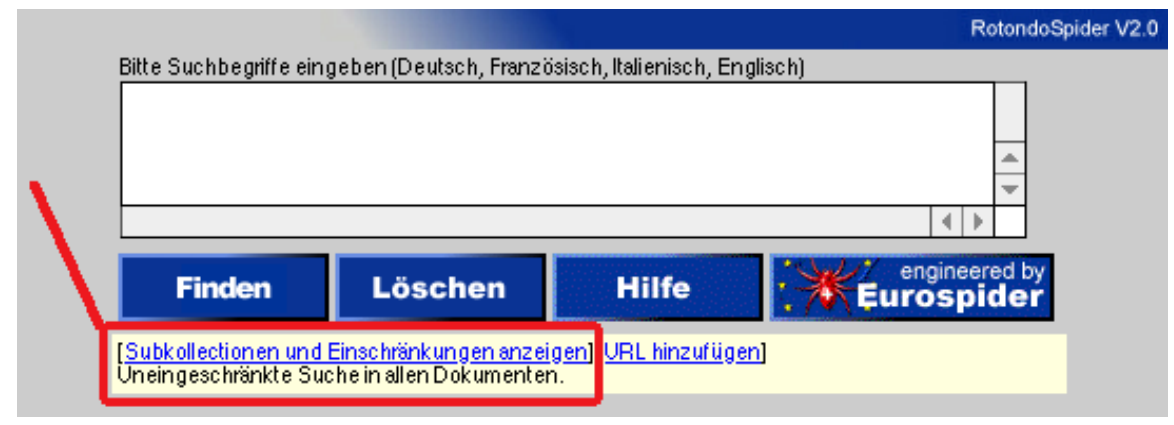

Ein Klick auf den Verweis "Subkollektionen und Einschränkungen anzeigen" auf der Suchmaske gerade unterhalb des "Finden"-Knopfes genügt, um mittels Javascript eine Liste der vorhandenen Subkollektionen zu erhalten. Um die Suche auf eine bestimmte Subkollektion zu beschränken, wird die entsprechende Subkollektion durch Klicken der Checkbox auf der linken Seite markiert. 


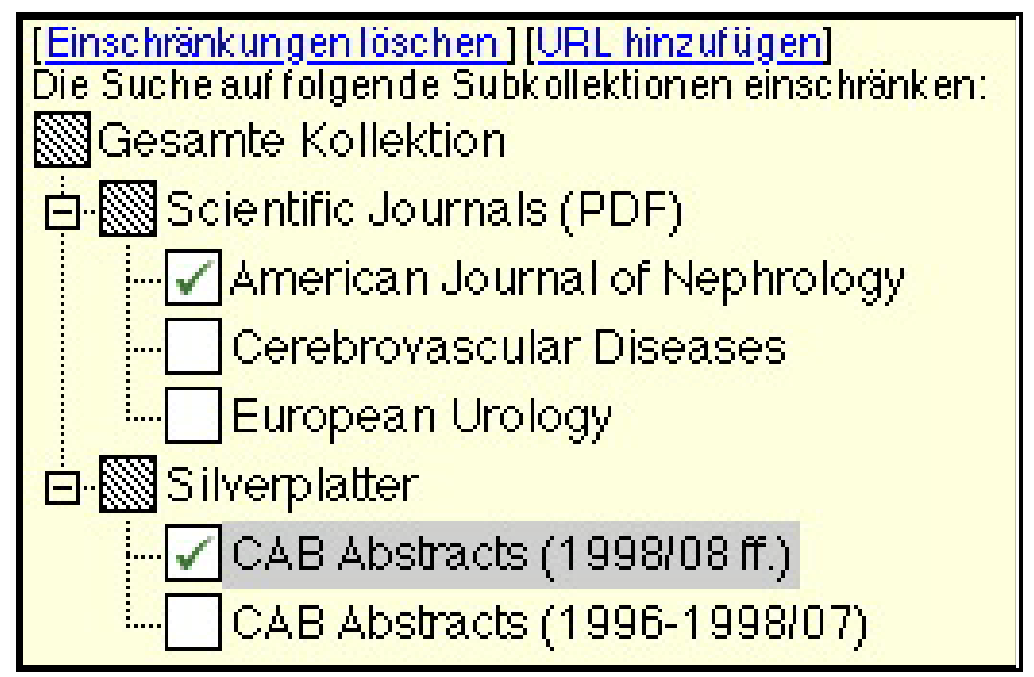

Auf diese Weise können auch mehrere Subkollektionen markiert werden.

\section{Spezielle Suchanfrage}

Neben der allgemeinen Suche in den Volltexten, ist auch eine spezielle Suche in bestimmten Datenfeldern möglich. Dies gilt natürlich nur dann, wenn der betreffende Datenbestand auch über derartige Spezifikationen verfügt. Ein Beispiel stellt die Suche in Silverplatter oder in dem OPAC dar, wo in den Feldern "Autorenfelder", "Titelfelder" und "Suche über alles" unterschieden ist.

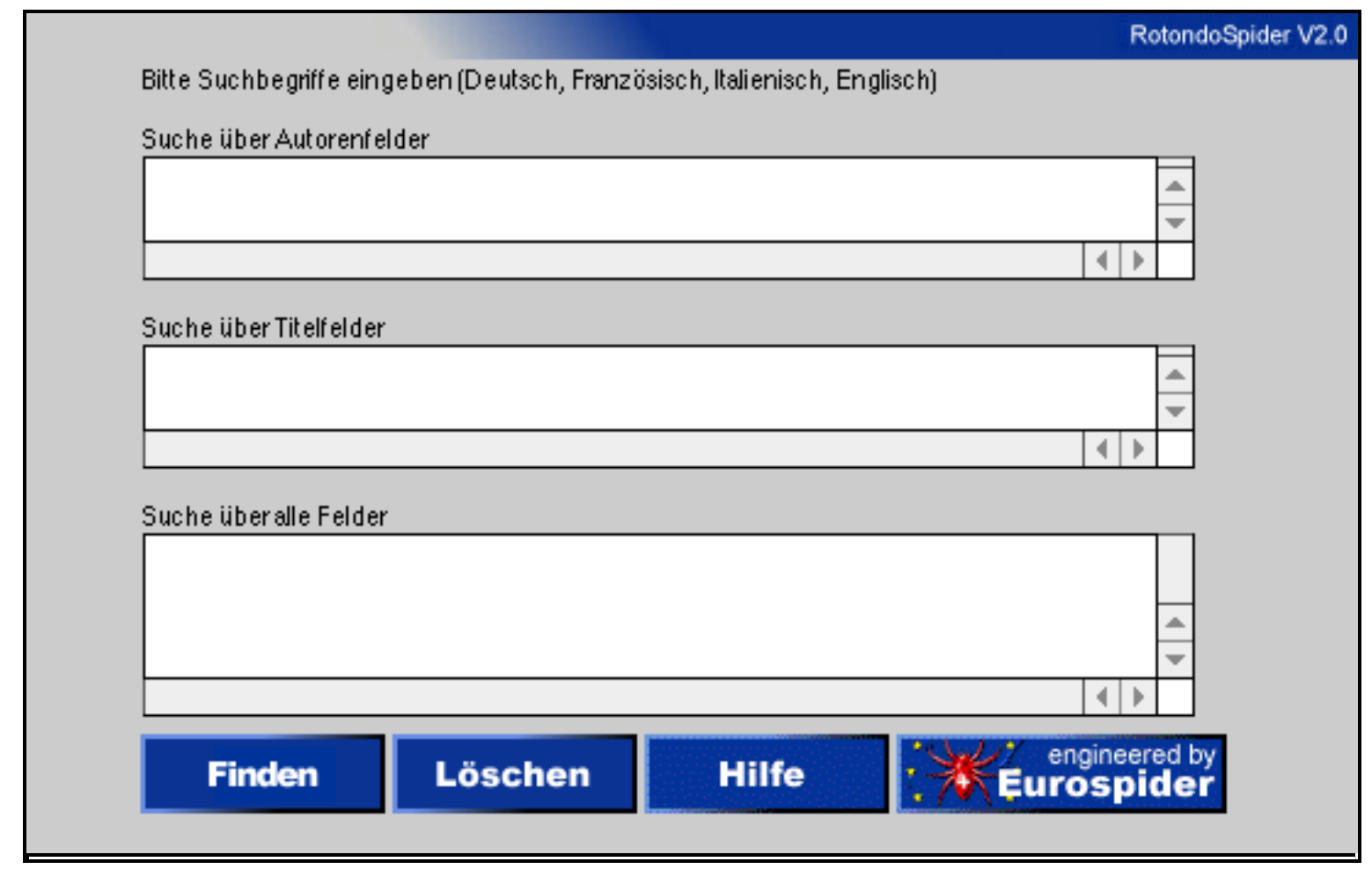

\section{Suchergebnis}

Das auf die Anfrage erzeugte Suchergebnis des Systems wird in einer Gesamt-Ergebnisliste präsentiert. Anhand der Farbgebung (Spektrum gelb-blau) wird vom System die entsprechende 
Wertung dargestellt. Das am ehesten zutreffende Objekt steht stets am oberen Ende der Liste. Dargestellt werden jeweils 10 Suchergebnisse, die übrigen lassen sich in 10-er-Schritten anzeigen, wenn die nach unten zeigenden Pfeile am Ende der Ergebnisliste geklickt werden.

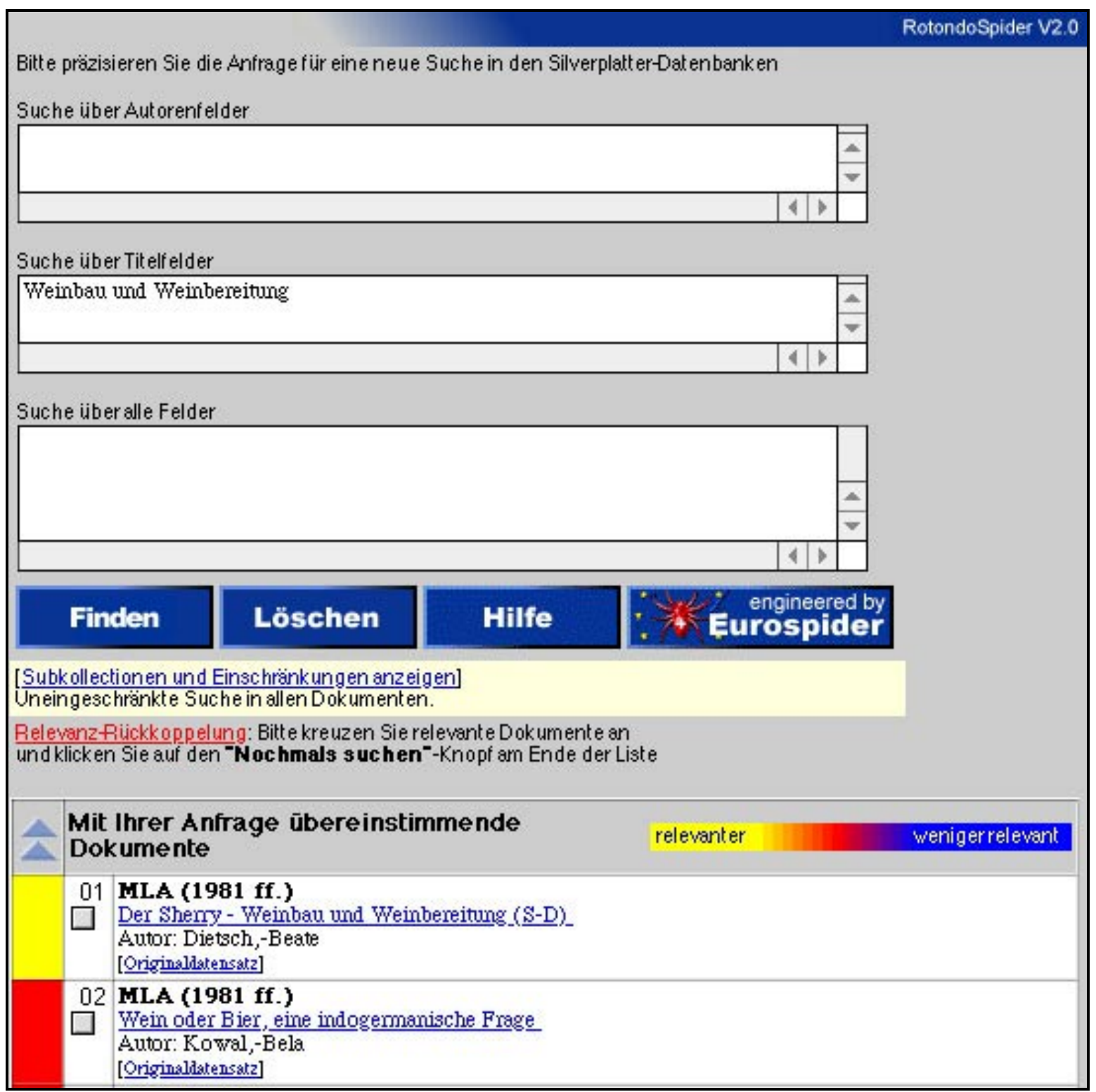

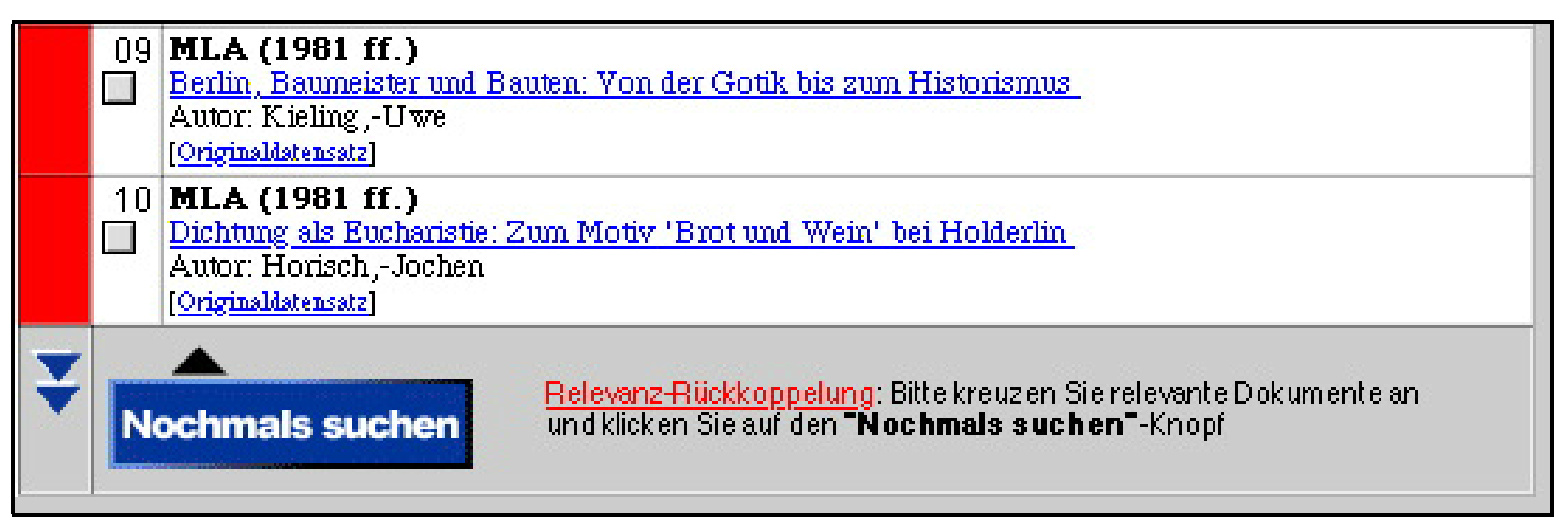




\section{Relevanz-Ranking}

Ein interessantes Feature stellt die Relevanz-Rückkopplung dar, die in das System der DigiBibHessen eingebaut ist. Wenn mehrere Dokumente in der Rangliste durchgesehen und einige davon als relevant eingestuft wurden, kann eine zweite verbesserte Suche gestartet werden. Dazu werden in der Rangliste die für relevant gehaltenen Dokumente in der Checkbox links neben den Ranglisten-Nummern markiert und anschließend der "Nochmals suchen"-Knopf am Fuss der Rangliste aktiviert. Die markierten Dokumente werden jetzt für die Suche verwandt. Diese Methode wird als Relevanz-Rückkopplung bezeichnet.

\section{Upload}

In einer digitalen Bibliothek ist die Abgabe von Dokumenten ebenso wie die Suche nach Dokumenten möglich. Um dem derzeit weitestgehend akzeptierten Standard bei der Kategorisierung von digitalen Dokumenten zu entsprechen, wurden die entsprechenden Abgabeseiten der DigiBib-Hessen gemäß Dublin-Core-Konventionen gestaltet. Die Autoren, die über die jeweilige DigiBib-Seite ihre Online-Publikationen abgeben wollen, können dies unter dem Punkt "Upload" tun.

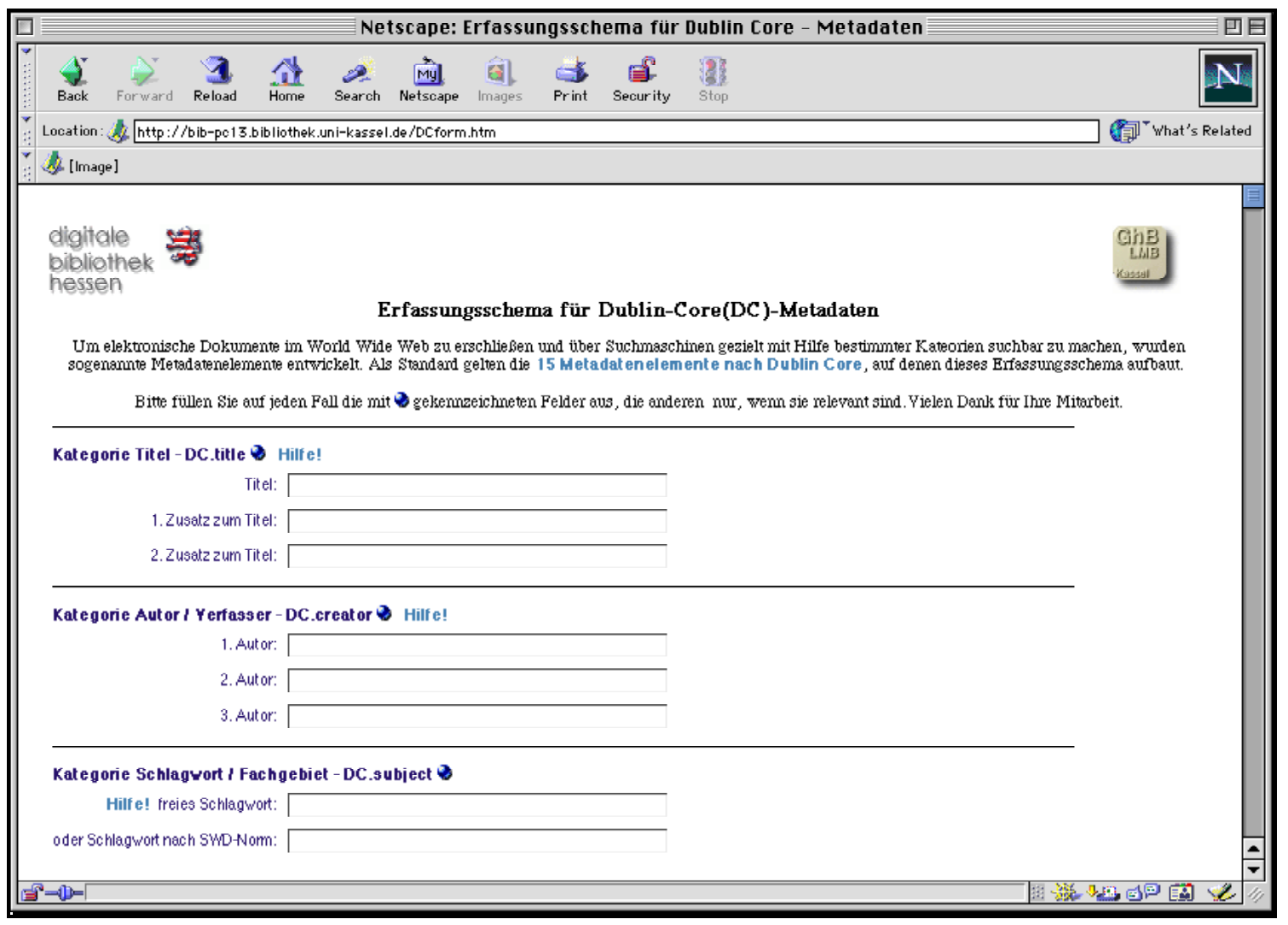

\section{Forum}

Um den Besuchern und Benutzern der DigiBib-Hessen die Gelegenheit zu geben, untereinander zu kommunizieren, wurde ein Forum eingerichtet. In diesem Forum können Nachrichten und Meinungen hinterlegt und gegebenenfalls beantwortet werden.

\section{News, Links, Impressum, Hilfe}

Entsprechend dem Forum für Benutzer ist gleichsam ein News-Board eingerichtet worden, in dem die Neuigkeiten (neue Dokumentlieferungen, neue Software-Features, etc.) zur DigiBib abrufbereit verzeichnet stehen. Unter den Punkten "Links" und "Impressum" sind weiterführende 
URL's bzw. das Projektteam benannt. Zu den einzelnen Funktionen der DigiBib-Hessen wurden Hilfetexte verfaßt, die in separat zu öffnenden Browserfenstern nachzulesen sind.

\section{Administrations-Tool}

Zur DigiBib-Hessen wurde ein Administrations-Tool entwickelt, mit dem das Gesamtsystem in grundlegenden Funktionen gesteuert und verwaltet werden kann. Auf diese Weise lassen sich beispielsweise neue digitale Dokumentgruppen neu eintragen resp. gruppieren. Dieses Administration-Tool hat weiterhin die Funktion, Prozesse zu überwachen, Statusberichte zu erzeugen und Statistiken zu erstellen.

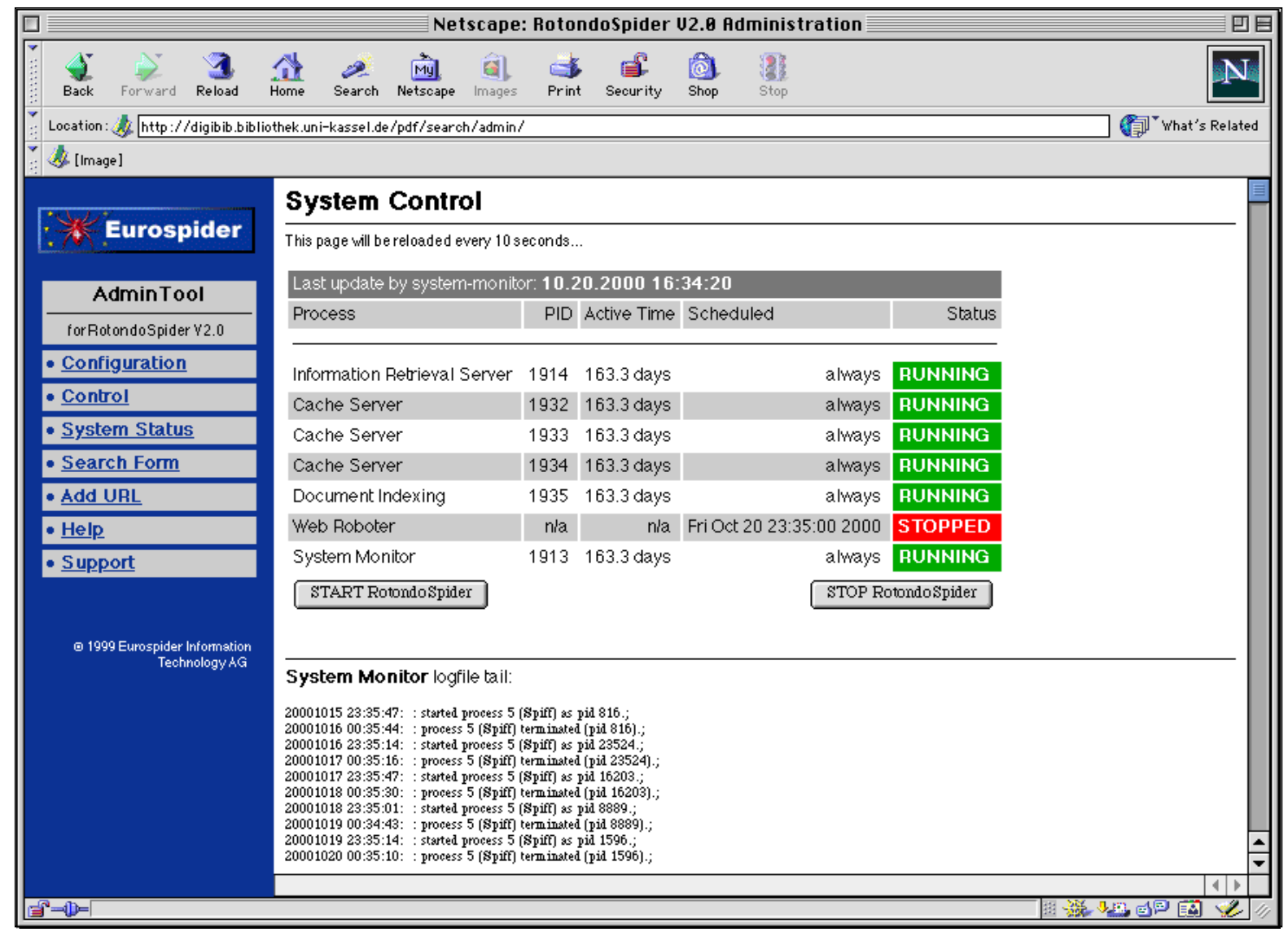

\section{Einbindung weiterer Bibliotheken}

Neben den technischen Weiterentwicklungen, die diese Testinstallation noch nehmen soll, ist ebenso eine Weiterentwicklung durch den Anschluß anderer Bibliotheken denkbar und konzeptuell unproblematisch möglich. Allen Beteiligten gleich wären die mit landesweiten Mitteln erworbenen Datenbanken und digitalen Dokumente. Jede Bibliothek würde zusätzlich über eine eigene zugeordnete Bibliothekssicht verfügen, so daß zusätzliche Dokumente, die nur für diesen Standort erworben und rechtlich zugeordet wurden, von den eigenen Benutzern gesehen und recherchiert werden könnten. Dabei ist nebensächlich, ob diese Dokumente auf einem zentralen Dokumentenserver oder auf dezentralen Servern abgelegt sind.

Innerhalb der Konzeption der DigiBib-Hessen ist der Benutzerauthentifizierungsprozeß über das LDAP-Protokoll auszuführen. Wie zuvor bemerkt, sollten an den jeweiligen Hochschulstandorten entsprechende LDAP-Server eingerichtet sein, die dann in ein Gesamtsystem eingebunden sind, jedoch inhaltlich nur vom jeweiligen Betreiber (HRZ) administriert werden. In diese LDAP-Server lassen sich zudem andere Funktionen (Chip-Karte) integrieren. 


\begin{abstract}
Ausblick
Derzeit ist nur ein Teilbestand der über landesweite Maßnahmen erworbenen Archivbestände von digitalen Dokumenten enthalten. Sofern die Verlage zügig liefern, wird sich in absehbarer Zeit ein erheblicher Zuwachs an Daten und Indizierungsmerkmalen ergeben. Diese Dokumente sind mit dieser Oberfläche komfortabel und genau suchbar. Letztlich wird allein im Laufe des Jahres 2000 durch die letztjährigen landesweiten Maßnahmen ein Bestand von 1100 elektronischen Zeitschriften, die teilweise bis 1995 zurückreichen, recherchierbar sein. Dies bedeutet allein einen Bestand von fast 180.000 Volltexten im PDF-Format. Ein Ausbau der DigiBib-Hessen ergibt gegenüber einer dezentralen Lagerung und Speicherung dieser Daten an allen Hochschulstandorten neben den Einsparungen bei Personal- und Sachkosten auch Einsparpotential bei den Verhandlungen weiterer Verträge.

Verglichen mit den jeweiligen Verlagsservern resp. -diensten kann man darauf hinweisen, daß bei dem meisten dieser Dienste bislang keine Volltextsuche in den eigenen PDF-Produkten möglich ist. In einem System wie der Digibib-Hessen kann zudem verlagsübergreifend ein Begriff in den Volltexten gesucht werden, da die Bestände indiziert vorliegen.
\end{abstract}

\title{
5.3 Zukünftige Vorhaben
}

\section{Teilprojekt: PDF}

Um die Suche bei langen digitalen Texten (Dissertationen) möglichst bequem zu gestalten, muß in dem aufgerufenen PDF-File der Such-Begriff farblich markiert sein (Highlighting in PDF's). Gleichzeitig sollte nur die betreffende Seite übertragen werden, um zusätzliches Scrollen und unnötige Netzlast zu vermeiden.

\section{Teilprojekt: Bildarchiv}

Anhand des Hessen-Media-Projektes "Kolonialbildarchiv" kann die vorhandene Funktionalität der Einbindung von Bilddaten gezeigt werden.

\section{Teilprojekt: Monitoring von Forschungsaktivitäten}

Anhand von ausgewählten Wissenschaftsdisziplinen sollte ein Monitoring von Forschungsaktivitäten in dem betreffenden Fachgebieten möglich sein. Externe PrePrint- und Forschungsserver müßten dazu in einem festen zeitlichen Rhythmus indiziert und mittels AgentSoftware befragt werden können. Auf diese Weise würde ein Nutzer einzig eine Suchanfrage an den Server stellen und z.B. per E-Mail benachrichtigt, wenn sich auf seinen Suchbegriff neue Informationen ergeben haben.

\section{Teilprojekt: Cross-Linguale Suche}

Neben der bereits realisierten multilingualen Suche mit multilingualen Indizes könnte ein crosslinguales Retrieval auf Dokumente bestimmter Fachbereiche (z.B. Medizin) geschaffen werden.

\section{Teilprojekt: Kostenpflichtiges Retrieval}

Für mögliche kostenpflichtige Dokumente resp. Informationen wird ein Abrechnungsmodul benötigt, bei dem die Anfragen pro User aufgezeichnet werden und den Dokumenten "Preise" zugewiesen werden können. Die Abrechnung kann dann durch das Stellen einer Rechnung geschehen. Echtes E-Commerce sollte solange aufgeschoben werden, bis sich eine entsprechende Lösung auf dem Markt durchgesetzt hat.

\section{Teilprojekt: Erschliessung von Multimedia-Dokumenten}

Neben Text- und Grafik-Retrieval soll weiter auch das Sprach-Retrieval in die "DigiBib-Hessen" einbezogen werden. 


\section{Teilprojekt: Digitalisierte Zettelkataloge}

Durch die vorhande TiFF-2-GIF-Konvertierung wäre eine Möglichkeit gegeben, die gescannten Kataloge mit in die Abfrage der "DigiBib-Hessen" einzubinden.

\section{Teilprojekt: Bestellmodul}

Die Anbindung an die PICA-Ausleihe/-Fernleihe ist unabdingbar für eine Weiterentwicklung und -Nutzung. Durch die Scriptingfunktionen in LBS 4 ist eine akzeptable Lösung für dieses Problem in Sicht.

\section{Teilprojekt: Mirroring und Load-Monitoring}

Es ist geplant, eine Online-Spiegelung der Indizes zwischen zwei Installationen aufzubauen, die Abschätzung der Last der beiden IR-Server im ServerMux vorzunehmen und entsprechend dem jeweiligen Load die Anfragen entsprechend zu verteilen.

\section{Ausblick}

Die Digib Hessen stellt,wie betont, einen Baustein zur Digitalen Bibliothek dar. Sie ist der Versuch, eine einheitliche Benutzeroberfläche über mehrere inhomogene Datenbanken mit Metadaten und Volltexten zu legen. Sie ist modular angelegt, beinhaltet eine DC-konforme Abgabemöglichkeit für digitale Medien und kann jederzeit durch weitere Komponenten oder Scripte erweitert werden. Es wäre wünschenswert, wenn sich andere Initiativen und Betreiber möglichst unter Hintanstellung kommerzieller oder politischer Interessen - zu einer Vorgehensweise entschließen könnten, die alle solchen Bausteine, die derzeit in Deutschland vorhanden sind, so zu gestalten, daß sie miteinander modular verbindbar oder zumindest kompatibel sind.

Die möglichen Umstände und Konsequenzen, die zu der Nutzung elektronischer Medien führen und bei der Nutzung elektronischer Medien entstehen, stecken erst in den Anfängen. Es nutzt wenig, Mißstände zu beklagen, sondern man muß sich vor allem klar machen, daß sich die Situation auch im Bibliothekssektor drastisch (weiter) entwickeln wird. Entscheidend für positive oder negative Entwicklungen wird dabei das Verständnis und die Fähigkeit sein, kompetent adäquate, am Nutzer orientierte Dienstleistungen anzubieten. Bereits heute ist Realität, daß eine reine Hol-Bibliothek, in der der Nutzer seine Bücher vorfindet oder mehr oder minder zeitintensiv bestellen kann, nicht mehr von allen Nutzern akzeptiert wird. Sicherlich wird auch die sog. "digitale Revolution" nicht sofort eine gänzlich andere Situation schaffen, jedoch dürfte die Entwicklung für Bibliotheken deutlich in eine Bring-Bibliothek gehen, also eine Bibliothek, die dem Nutzer die benötigten Informationen schnell und kostengünstig bereits am jeweiligen Arbeitsplatz zur Verfügung stellt. 